\title{
The blow-up curve of solutions of mixed problems for semilinear wave equations with exponential nonlinearities in one space dimension, II
}

\author{
by

\section{Paul GODIN ${ }^{1}$} \\ Université Libre de Bruxelles, Département de Mathématiques, Campus Plaine CP 214, \\ Boulevard du Triomphe, B-1050 Bruxelles, Belgium \\ Manuscript received 28 February 2000
}

ABSTRACT. - In this paper, we consider mixed problems with a timelike boundary derivative (or a Dirichlet) condition for semilinear wave equations with exponential nonlinearities in a quarter plane. The case when the boundary vector field is tangent to the characteristic which leaves the domain in the future is also considered. We show that solutions either are global or blow up on a $C^{1}$ curve which is spacelike except at the point where it meets the boundary; at that point, it is tangent to the characteristic which leaves the domain in the future.

(C) 2000 L'Association Publications de l'Institut Henri Poincaré. Published by Elsevier B.V. All rights reserved

Key words: Semilinear wave equations, Mixed problems, Blow-up of solutions

RÉSUMÉ. - Dans cet article, nous considérons des problèmes mixtes avec une condition au bord de type temps (ou de Dirichlet) pour des équations d'ondes semi-linéaires à non-linéarités exponentielles dans un quart de plan. Le cas où le champ de vecteurs au bord est tangent à la caractéristique qui quitte le domaine dans le futur est aussi considéré. Nous montrons que les solutions soit sont globales, soit explosent (au moins hors du bord) sur une courbe $C^{1}$ qui est orientée d'espace sauf

\footnotetext{
${ }^{1}$ E-mail: pgodin@ulb.ac.be.
} 
au point où elle rencontre le bord; en ce point, elle est tangente à la caractéristique qui quitte le domaine dans le futur.

(C) 2000 L'Association Publications de 1 Institut Henri Poincaré. Published by Elsevier B.V. All rights reserved

Mots Clés: Équations d'ondes semi-linéaires, Problèmes mixtes, Explosion des solutions

\section{INTRODUCTION}

This paper is a continuation of [3] in which a study was made of the blow-up curve of solutions of mixed problems in a quarter plane for semilinear wave equations with exponential nonlinearities, when the boundary vector field is spacelike. In that case, results similar to those of Caffarelli and Friedman [1,2] were obtained: it was proved in [3] that non global solutions blow up on a $C^{1}$ spacelike curve. When the boundary vector field is tangent to the characteristic which leaves the domain in the future, weaker results were obtained in [3]. In the present paper, we consider the case when the boundary vector field is constant and either timelike or tangent to the characteristic which leaves the domain in the future; we also consider the case of a Dirichlet boundary condition. The blow-up method of [1,2], adapted in [3], does not seem to be easily applicable in the present situation. We impose conditions on the nonlinearities which are more restrictive than those of [3]. By a completely different method based on conservation laws, we show that non global solutions still blow up (in a sense which will be made clear) on a $C^{1}$ curve which is now spacelike except at the meeting point with the boundary where it is characteristic (actually tangent to the characteristic which leaves the domain in the future).

Our paper is organized as follows. In Section 2 we recall some results of [3] and state our new results precisely. In Section 3, we show that a certain result of [3] is impossible if the boundary vector field is timelike. Asymptotic expansions are obtained in Section 4, and the proofs are completed in Section 5. In order to avoid interruptions in a number of proofs, some useful results on mixed problems and on fundamental systems of solutions of ordinary differential equations are collected in two appendices at the end of the paper: in particular, some estimates from [3] are recalled in the first appendix. 


\section{STATEMENT OF THE RESULTS}

We consider the following mixed problem for (real-valued) functions $u$ of $(x, t)$ :

$$
\begin{gathered}
\square u=F(u) \quad \text { if } x>0 \text { and } t>0, \\
u_{x}+\gamma u_{t}=0 \quad \text { if } x=0 \text { and } t>0, \\
u=\psi_{0} \quad \text { and } \quad u_{t}=\psi_{1} \quad \text { if } x>0 \text { and } t=0,
\end{gathered}
$$

where $\square=\partial_{t}^{2}-\partial_{x}^{2}$ and $\gamma \neq 1,|\gamma| \geqslant 1$ (the case $|\gamma|<1$ has been considered in [3]). We introduce the compatibility conditions

$$
\begin{gathered}
\psi_{0}^{\prime}(0)+\gamma \psi_{1}(0)=0, \\
\psi_{1}^{\prime}(0)+\gamma\left(\psi_{0}^{\prime \prime}(0)+F\left(\psi_{0}(0)\right)\right)=0, \\
\psi_{0}^{\prime \prime \prime}(0)+\gamma \psi_{1}^{\prime \prime}(0)=0 .
\end{gathered}
$$

The following result is well known. A proof has been given in the appendix of [3]. We write $\mathbb{R}^{+}=\{s \in \mathbb{R}, s>0\}, \overline{\mathbb{R}^{+}}=\{s \in \mathbb{R}, s \geqslant 0\}$.

THEOREM 2.1. -

(1) If $F \in C^{1}(\mathbb{R}), \gamma \neq 1, \psi_{j} \in C^{2-j}\left(\overline{\mathbb{R}^{+}}\right)$for $j=0,1$, and if (2.4), (2.5) are satisfied, there exists an open neighborhood $U$ of $\{0\} \times$ $\overline{\mathbb{R}^{+}}$in $\left(\overline{\mathbb{R}^{+}}\right)^{2}$ such that $(2.1),(2.2),(2.3)$ has exactly one solution $u \in C^{2}(\bar{U})$.

(2) If furthermore $F \in C^{2}(\mathbb{R}), \psi_{j} \in C^{3-j}\left(\overline{\mathbb{R}^{+}}\right)$for $j=0,1$ and (2.6) holds, then the conclusion of (1) still holds with some $u \in C^{3}(\bar{U})$.

(3) If furthermore $F \in L^{\infty}(\mathbb{R})$ in (1) or (2), one can take $U=\left(\overline{\mathbb{R}^{+}}\right)^{2}$.

If $\gamma=1$, Theorem 2.1 is already false when $F \equiv 0$. In [1,2], Caffarelli and Friedman have studied the blow-up of solutions of Cauchy problems for equations of type (2.1), when $F(u)$ is bounded below on $\mathbb{R}$ and behaves like $u^{p}, p>1$, as $u \rightarrow+\infty$. In [3] we have assumed that $F$ satisfies the following conditions.

$F \in C^{2}(\mathbb{R})$ and for some $C_{0}, C_{1}, C_{2}, p, A>0$, the following holds:

(1) $F(z) \geqslant-C_{0}$ if $z \in \mathbb{R}$;

(2) $\mathrm{e}^{-p z} F(z) \rightarrow A$ as $z \rightarrow+\infty$;

(3) $C_{1} \leqslant \mathrm{e}^{-p z} F^{\prime}(z) \leqslant C_{2}$ if $z>0$ is large;

(4) $\left|F^{\prime \prime}(z)\right| \leqslant C_{2} \mathrm{e}^{p z}$ if $z>0$. 
Under the assumption (2.7), we have considered in [3] the Cauchy problem

$$
\begin{gathered}
\square u=F(u) \quad \text { if } x \in \mathbb{R} \text { and } t>0, \\
u=u_{0} \quad \text { and } \quad u_{t}=u_{1} \quad \text { if } x \in \mathbb{R} \text { and } t=0,
\end{gathered}
$$

where $u_{j} \in C^{3-j}(\mathbb{R})$ (and $u_{j}$ is real-valued), $j=0,1$. We have proved:

THEOREM 2.2 (cf. [3]). - Assume that (2.7) holds. Then there exist

(1) $\varphi: \mathbb{R} \rightarrow] 0,+\infty]$ such that $\varphi(\mathbb{R}) \subset \mathbb{R}^{+}$or $\varphi(\mathbb{R})=\{+\infty\}$,

(2) $u \in C^{3}(\Omega)$, where $\Omega=\left\{(x, t) \in \mathbb{R}^{2}, 0 \leqslant t<\varphi(x)\right\}$, such that $u$ is a solution of (2.8), (2.9) in $\Omega$,

with the following properties: if $\varphi(\mathbb{R}) \subset \mathbb{R}^{+}$, then $\varphi \in C^{1}(\mathbb{R}),\left|\varphi^{\prime}(x)\right|<1$ for all $x \in \mathbb{R}$, and $u(y, s) \rightarrow+\infty$ if $s<\varphi(y)$ and $(y, s) \rightarrow(x, \varphi(x))$ for some $x \in \mathbb{R}$.

The next two theorems have also been proved in [3].

THEOREM 2.3. - Assume that (2.4), (2.5), (2.6), (2.7) hold and that $|\gamma|<1$. Then there exist

(1) $\left.\left.\varphi: \overline{\mathbb{R}^{+}} \rightarrow\right] 0,+\infty\right]$ such that $\varphi\left(\overline{\mathbb{R}^{+}}\right) \subset \mathbb{R}^{+}$or $\varphi\left(\overline{\mathbb{R}^{+}}\right)=\{+\infty\}$,

(2) $u \in C^{3}(\Omega)$, where $\Omega=\left\{(x, t) \in\left(\overline{\mathbb{R}^{+}}\right)^{2}, t<\varphi(x)\right\}$, such that $u$ is a solution of (2.1), (2.2), (2.3) if $(x, t) \in \Omega$,

with the following properties: if $\varphi\left(\overline{\mathbb{R}^{+}}\right) \subset \mathbb{R}^{+}$, then $\varphi \in C^{1}\left(\overline{\mathbb{R}^{+}}\right),\left|\varphi^{\prime}(x)\right|$ $<1$ for all $x \in \overline{\mathbb{R}^{+}}, \varphi^{\prime}(0)=\gamma$ and $u(y, s) \rightarrow+\infty$ if $s<\varphi(y)$ and $(y, s) \rightarrow(x, \varphi(x))$ for some $x \in \overline{\mathbb{R}^{+}}$.

THEOREM 2.4. - Assume that (2.4), (2.5), (2.6), (2.7) hold and that $\gamma=-1$. Then there exist

(1) $\left.\left.\varphi: \overline{\mathbb{R}^{+}} \rightarrow\right] 0,+\infty\right]$ such that $\varphi\left(\overline{\mathbb{R}^{+}}\right) \subset \mathbb{R}^{+}$or $\varphi\left(\overline{\mathbb{R}^{+}}\right)=\{+\infty\}$,

(2) $u \in C^{3}(\Omega)$, where $\Omega=\left\{(x, t) \in\left(\overline{\mathbb{R}^{+}}\right)^{2}, t<\varphi(x)\right\}$ such that $u$ is a solution of (2.1), (2.2), (2.3) if $(x, t) \in \Omega$,

with the following properties: if $\varphi\left(\overline{\mathbb{R}^{+}}\right) \subset \mathbb{R}^{+}$, then $\varphi \in C^{1}\left(\mathbb{R}^{+}\right), u(y, s)$ $\rightarrow+\infty$ if $s<\varphi(y)$ and $(y, s) \rightarrow(x, \varphi(x))$ for some $x \in \overline{\mathbb{R}^{+}}$, and for each $R>0$, one can find $\theta \in] 0,1\left[\right.$ such that $-1<\varphi^{\prime}(x)<\theta$ if $0<x<R$.

To improve the results of [3] when $\gamma=-1$, and to study the cases when $|\gamma|>1$ or the Dirichlet boundary condition, we shall have to use a different method and make more restrictive assumptions on $F$. 
We shall consider the following additional assumption on $F$ (with $A, p$ as in (2.7)):

One can find $C>0, \alpha<\frac{1}{2}$ such that $\left|F(z)-A \mathrm{e}^{p z}\right| \leqslant C \mathrm{e}^{\alpha p z}$ if $z \geqslant 0$.

Then we have the following result, which is similar to Theorem 2.3.

THEOREM 2.5. - Let all assumptions of Theorem 2.4 hold, and assume moreover that (2.10) is satisfied. Then we have the following additional conclusion: if $\varphi\left(\overline{\mathbb{R}^{+}}\right) \subset \mathbb{R}^{+}$, then $\varphi \in C^{1}\left(\overline{\mathbb{R}^{+}}\right)$and $\varphi^{\prime}(0)=-1$.

For the sequel, and also to compare Theorem 2.5 with Theorem 2.8 below, it will be convenient to reformulate (2.10) in the following way:

$$
\begin{aligned}
& \text { If } g \in C^{2}\left(\overline{\mathbb{R}^{+}}\right) \text {satisfies the equation } g^{\prime}=p F-F^{\prime}, \\
& \text { there exist } C>0, \alpha<\frac{1}{2} \text { such that }|g(z)| \leqslant C \mathrm{e}^{\alpha p z} \text { if } z \geqslant 0 \text {. }
\end{aligned}
$$

Indeed, if (2.10) holds, integration of the relation $g^{\prime}(t)=p(F(t)-$ $\left.A \mathrm{e}^{p t}\right)-\left(F(t)-A \mathrm{e}^{p t}\right)^{\prime}$ over $[0, z]$ yields (2.11) (with a larger $\alpha$ if $\alpha \leqslant 0)$. And if (2.11) holds, integration of the relation $\left(\mathrm{e}^{-p t}(F+\right.$ $g)(t))^{\prime}=-p \mathrm{e}^{-p t} g(t)$ over $[0, z]$ shows that $\lim _{z \rightarrow+\infty} \mathrm{e}^{-p z} F(z)$ exists, and integration of the same relation over $[z,+\infty[$ then yields $(2.10)$ (with some $A \in \mathbb{R}$ ).

The following simple theorem shows that when $|\gamma|>1$ and the solution blows up on a $C^{1}$ curve $t=\varphi(x)$, we cannot expect to have $\varphi^{\prime}(0)=\gamma$ as in Theorems 2.3 and 2.5.

THEOREM 2.6. - Assume that $x_{0} \geqslant 0$, that $V$ is an open neighborhood of $x_{0}$ in $\overline{\mathbb{R}^{+}}$, that $\varphi: V \rightarrow \mathbb{R}^{+}$and that $\Omega=\left\{(x, t) \in V \times \mathbb{R}^{+}, t<\right.$ $\varphi(x)\}$ is open. Assume that $\square u=F(u)$ in $\Omega$, where $u \in C^{2}(\Omega)$ and $F$ is bounded below on $\mathbb{R}$ and bounded above on every half line $]-\infty, a[, a \in$ $\mathbb{R}$. Assume that $|u(y, s)| \rightarrow+\infty$ as $s<\varphi(y)$ and $(y, s) \rightarrow\left(x_{0}, \varphi\left(x_{0}\right)\right)$. Then

(1) one can find a sequence $\left(x_{k}\right)$ with $x_{k} \rightarrow x_{0}$ and $\varphi\left(x_{k}\right)-\varphi\left(x_{0}\right) \leqslant$ $x_{k}-x_{0}$

(2) if furthermore $x_{0}>0$, one can find a sequence $\left(y_{k}\right)$ with $0<y_{k} \stackrel{<}{\rightarrow}$ $x_{0}$ and $\varphi\left(x_{0}\right)-\varphi\left(y_{k}\right) \geqslant-x_{0}+y_{k}$. 
Remark 2.1. - Theorem 2.6 is applicable if $F(u)=\mathrm{e}^{u}$. However its conclusions are false if $F(u)=u^{p}, p \in 2 \mathbb{N} \backslash\{0\}$, as the function $u(x, t)=a(t-\gamma x-1)^{-q}\left(\right.$ where $a \in \mathbb{R}$ and $a^{p-1}=q(q+1)\left(1-\gamma^{2}\right)$, $\left.q=\frac{2}{p-1}\right)$ shows. If $\gamma>1$, this function is a solution of a problem of type (2.1), (2.2), (2.3) when $0<t<\gamma x+1, x>0$.

Remark 2.2. - The conclusions of Theorem 2.6 are false for $F(u)=$ $\mathrm{e}^{u}$ if we consider complex-valued solutions, as the example $u(x, t)=$ $\ln \frac{2\left(1-\gamma^{2}\right)}{(t-\gamma x-1)^{2}},|\gamma|>1$ shows.

We shall also consider the Dirichlet boundary condition

$$
u=0 \quad \text { if } x=0 \text { and } t>0,
$$

and introduce the corresponding compatibility conditions for (2.1), (2.3), namely

$$
\begin{aligned}
& \psi_{0}(0)=0, \\
& \psi_{1}(0)=0, \\
& \psi_{0}^{\prime \prime}(0)+F(0)=0, \\
& \psi_{1}^{\prime \prime}(0)=0 .
\end{aligned}
$$

The following well known result corresponds to Theorem 2.1.

THEOREM 2.7. - Theorem 2.1 remains true if (2.2), (2.4), (2.5), (2.6) are replaced by (2.12)-(2.16).

The proof of Theorem 2.1 given in [3] can easily be modified to give a proof of Theorem 2.7. We omit the details.

When $|\gamma|>1$ or when (2.2) is replaced by (2.12), the method of proof of Theorem 2.3 does not seem to work. However we can still prove the existence of a blow-up curve if we add the following rather restrictive assumptions on the nonlinearity $F(u)$ :

(1) $F \in L^{\infty}\left(\mathbb{R}^{-}\right)$in case $\gamma>1$;

(2) if $g \in C^{2}(\mathbb{R})$ satisfies the equation $g^{\prime}=p F-F^{\prime}$ on $\mathbb{R}$, then $g, g^{\prime} \in L^{\infty}(\mathbb{R})$ in case $\gamma>1$, and $g, g^{\prime} \in L^{\infty}\left(\mathbb{R}^{+}\right)$in case $\gamma<-1$ or when the boundary condition is given by (2.12).

We shall prove the following result. 
THEOREM 2.8. - (I) Assume that $|\gamma|>1$, and that (2.4), (2.5), (2.6), (2.7), (2.17) hold. Then the conclusions of Theorem 2.3 still hold with the following modifications: if $\varphi\left(\overline{\mathbb{R}^{+}}\right) \subset \mathbb{R}^{+}$, now $\left|\varphi^{\prime}(x)\right|<1$ only if $x>0$, $\varphi^{\prime}(0)=-1$, and $u(y, s) \rightarrow+\infty$ if $s<\varphi(y)$ and $(y, s) \rightarrow(x, \varphi(x))$ for some $x$, where $x>0$ if $\gamma>1$ and $x \geqslant 0$ if $\gamma<-1$.

(II) Assume that (2.13), (2.14), (2.15), (2.16), (2.7), (2.17) hold. Then the conclusions of (I) for $\gamma>1$ still hold for the problem (2.1), (2.12), (2.3).

Remark 2.3. - The function $u(x, t)=\ln \left(2 / \cosh ^{2} x\right)$ satisfies $\square u=\mathrm{e}^{u}$ if $(x, t) \in \mathbb{R}^{2}$; furthermore, for all $\gamma \in \mathbb{R}, u_{x}+\gamma u_{t}=0$ if $x=0$, and $u=0$ if $x=\ln (\sqrt{2}+1)$. Hence it may happen that $\varphi \equiv+\infty$ in Theorems $2.2,2.3,2.4,2.5,2.8$.

Remark 2.4. - Assume that $F$ satisfies the assumptions of Theorem 2.2 and that $F \geqslant 0$. Denote by $\mathcal{T}$ the triangular domain with vertices $(a, 0),(b, 0),\left(\frac{a+b}{2}, \frac{b-a}{2}\right)$, where $0<a<b$. It follows from the results of [6] that one can find $\psi_{0}, \psi_{1} \in C^{\infty}([a, b])$ such that there is no $u \in C^{2}(\overline{\mathcal{T}})$ satisfying $\partial_{t}^{j} u=\psi_{j}$ in $] a, b[\times\{0\}$ for $j=0,1$ and $\square u=F(u)$ in $\mathcal{T}$. Extending $\psi_{0}, \psi_{1}$ to $\overline{\mathbb{R}^{+}}$in such a way that the compatibility conditions (2.4), (2.5), (2.6) (or (2.13), (2.14), (2.15), (2.16)) are satisfied, we obtain examples for Theorems $2.3,2.4,2.5,2.8$ with $\varphi\left(\overline{\mathbb{R}^{+}}\right) \subset \mathbb{R}^{+}$. When, e.g., $F(u)=\mathrm{e}^{u}$, see also [7] and references given there for constructions which yield examples for Theorem 2.3.

Remark 2.5. - Replacing $u(x, t)$ by $p u\left((A p)^{-1 / 2} x,(A p)^{-1 / 2} t\right)$ and $F(z)$ by $A^{-1} F\left(p^{-1} z\right)$, we may and shall assume in the rest of the paper that $p=A=1$ in (2.7), (2.10), (2.17). This will simplify a number of expressions later on.

Remark 2.6. - In Theorem 2.2, $\Omega$ is the maximal influence domain of $\mathbb{R} \times \overline{\mathbb{R}^{+}}$, containing $\mathbb{R} \times\{0\}$, in which (2.8), (2.9) has a (unique) $C^{3}$ solution. Likewise, in Theorems 2.3, 2.4, 2.5, 2.7, 2.8, $\Omega$ is the maximal influence domain of $\left(\overline{\mathbb{R}^{+}}\right)^{2}$, containing $\overline{\mathbb{R}^{+}} \times\{0\}$, in which (2.1), (2.2) (or (2.12)), (2.3) has a unique $C^{3}$ solution.

\section{BOUNDS FOR SOLUTIONS OF LINEAR DIRICHLET PROBLEMS}

If $R>0$, put $D_{R}=\left\{(x, t) \in\left(\mathbb{R}^{+}\right)^{2}, x+t<R\right\}$. If $(x, t) \in\left(\overline{\mathbb{R}^{+}}\right)^{2}$, write $K^{ \pm}(x, t)=\left\{(y, s) \in\left(\overline{\mathbb{R}^{+}}\right)^{2}, s \gtrless t,|y-x|<|t-s|\right\}$. Finally, if $(x, t) \in$ $\overline{D_{R}}$, put $K_{R}^{+}(x, t)=K^{+}(x, t) \cap \overline{D_{R}}$. Assume that $\psi: \overline{\mathbb{R}^{+}} \rightarrow \mathbb{R}^{+}$satisfies 
$\left|\psi\left(x_{1}\right)-\psi\left(x_{2}\right)\right| \leqslant\left|x_{1}-x_{2}\right|$ for all $x_{1}, x_{2} \geqslant 0$ and write $\mathcal{U}=\{(x, t) \in$ $\left.\left(\overline{\mathbb{R}^{+}}\right)^{2}, t<\psi(x)\right\}$. Assume that $u \in C^{2}\left(\mathcal{U} \cap \overline{D_{R}}\right), F \in C\left(\mathcal{U} \cap \overline{D_{R}}\right)$, and that the following holds:

$$
\begin{gathered}
\square u=F \quad \text { in } \mathcal{U} \cap D_{R}, \\
u=0 \quad \text { if } x=0 \text { and } 0<t<\min (\psi(0), R), \\
u=\psi_{0} \quad \text { and } \quad u_{t}=\psi_{1} \quad \text { if } 0<x<R \text { and } t=0 .
\end{gathered}
$$

It is certainly well known and easily checked by integration of $\square u$ over $K^{-}(x, t)$, and also over $K^{-}(0, t-x)$ if $x<t$, and by use of the divergence formula, that $u=u_{1}+u_{2}$, where

$$
\begin{aligned}
& u_{1}(x, t)=\frac{1}{2}\left(\psi_{0}(t+x)-\psi_{0}(t-x)\right)+\frac{1}{2} \int_{t-x}^{t+x} \psi_{1}(y) d y \\
& \text { if } x<t \text {, } \\
& u_{1}(x, t)=\frac{1}{2}\left(\psi_{0}(x+t)+\psi_{0}(x-t)\right)+\frac{1}{2} \int_{x-t}^{x+t} \psi_{1}(y) d y \\
& \text { if } x>t \text {. } \\
& u_{2}(x, t)=\frac{1}{2} \int_{t-x}^{t}\left(\int_{x-t+s}^{x+t-s} F(y, s) d y\right) d s \\
& +\frac{1}{2} \int_{0}^{t-x}\left(\int_{t-s-x}^{t-s+x} F(y, s) d y\right) d s \text { if } x<t, \\
& u_{2}(x, t)=\frac{1}{2} \int_{0}^{t}\left(\int_{x-t+s}^{x+t-s} F(y, s) d y\right) d s \quad \text { if } x>t .
\end{aligned}
$$

The following result immediately follows from (3.4), (3.5).

LEMMA 3.1. - If $C_{0}>0$ and $F \geqslant-C_{0}$, one can find $C>0$ (depending on $\psi_{0}, \psi_{1}, R, C_{0}$, but not on $F$ ) such that $u \geqslant-C$ in $\mathcal{U} \cap \overline{D_{R}}$. 


\section{PROOF OF THEOREM 2.6}

Let us prove (1). Assume that, for some $\varepsilon>0, \varphi(x)-\varphi\left(x_{0}\right)>x-x_{0}$ if $\left.x \in] x_{0}, x_{0}+\varepsilon\right]$. Assume that $\varepsilon<\varphi\left(x_{0}\right)$ and denote by $\mathcal{P}_{0}$ the triangular domain with vertices $\left(x_{0}, \varphi\left(x_{0}\right)\right),\left(x_{0}+\varepsilon, \varphi\left(x_{0}\right)-\varepsilon\right),\left(x_{0}+\varepsilon, \varphi\left(x_{0}\right)+\right.$ $\varepsilon)$. If $(x, t) \in \mathcal{P}_{0}$, denote by $\mathcal{P}$ the triangular domain with vertices $(x, t),\left(x_{0}+\varepsilon, t-x_{0}-\varepsilon+x\right),\left(x_{0}+\varepsilon, t+x_{0}+\varepsilon-x\right)$. We have

$$
\begin{aligned}
u(x, t)= & \frac{1}{2}\left(u\left(x_{0}+\varepsilon, t+x_{0}+\varepsilon-x\right)+u\left(x_{0}+\varepsilon, t-x_{0}-\varepsilon+x\right)\right) \\
& -\frac{1}{2} \int_{t-x_{0}-\varepsilon+x}^{t+x_{0}+\varepsilon-x} \partial_{1} u\left(x_{0}+\varepsilon, s\right) d s-\frac{1}{2} \iint_{\mathcal{P}} F(u)(y, s) d y d s .
\end{aligned}
$$

Since $F$ is bounded below, it follows from (4.1) that $u$ is bounded above in $\mathcal{P}_{0}$. But then (4.1) again shows that $u$ is bounded below in $\mathcal{P}_{0}$. This contradicts the fact that $|u(x, t)| \rightarrow \infty$ as $t<\varphi(x)$ and $(x, t) \rightarrow$ $\left(x_{0}, \varphi\left(x_{0}\right)\right)$. This proves (1). The proof of (2) is completely similar and may be omitted. The proof of Theorem 2.6 is complete.

\section{SOME ESTIMATES OF SOLUTIONS}

Our purpose is to prove the following two propositions, which will play an important role in the proof of Theorems 2.5 and 2.8. Recall that we assume, as we may, that $p=A=1$ in (2.7) (2), (2.10), (2.17) (see Remark 2.5). As before, we put $D_{R}=\left\{(x, t) \in\left(\mathbb{R}^{+}\right)^{2}, x+t<R\right\}$ if $R>0$.

Proposition 5.1. - Let $F \in C^{1}(\mathbb{R})$ satisfy (2.7) (1) and (2.17) (with $\left.g \in C^{1}(\mathbb{R})\right)$, and assume that $t_{0}>0$, that

$$
u \in C^{2}\left(\overline{D_{t_{0}}} \backslash\left\{\left(0, t_{0}\right)\right\}\right) \cap\left(\bigcap_{0<T<t_{0}} C^{3}\left(\overline{D_{T}}\right)\right)
$$

and that $\square u=F(u)$ in $D_{t_{0}}$.

(I) If $u_{x}+\gamma u_{t}=0$ when $x=0$ and $0 \leqslant t<t_{0}$ (where $\gamma \neq 1$ ) and if $t \mapsto u_{t}(0, t)$ is bounded when $0 \leqslant t<t_{0}$, it follows that $u \in C^{2}\left(\overline{D_{t_{0}}}\right)$.

(II) If $u=0$ when $x=0$ and $0 \leqslant t<t_{0}$ and if $t \mapsto u_{x}(0, t)$ is bounded when $0 \leqslant t<t_{0}$, it follows that $u \in C^{2}\left(\overline{D_{t_{0}}}\right)$. 
When, at the contrary, the function $t \mapsto\left(u_{x}(0, t), u_{t}(0, t)\right)$ is unbounded when $0 \leqslant t<t_{0}$, one can obtain asymptotic expansions when $x=0$, as the next proposition shows. Henceforth we shall write $\partial_{\xi}=$ $\partial_{t}+\partial_{x}, \partial_{\eta}=\partial_{t}-\partial_{x}, z_{\xi}=\partial_{\xi} z, z_{\eta}=\partial_{\eta} z$.

Proposition 5.2. - Let $\chi: \overline{\mathbb{R}^{+}} \rightarrow \mathbb{R}^{+}$be such that $\chi(0)=t_{0}>0$, $\chi(x) \geqslant t_{0}-x$. Put $\Lambda=\left\{(x, t) \in\left(\overline{\mathbb{R}^{+}}\right)^{2}, t<\chi(x)\right\}$. Let $F \in C^{1}(\mathbb{R})$ satisfy (2.7)(1), (2.7)(2), and assume that $u \in C^{3}(\Lambda), \square u=F(u)$ in $\Lambda$.

(I) If $F$ also satisfies (2.17) (with $g \in C^{1}(\mathbb{R})$ ), if $u_{x}+\gamma u_{t}=0$ when $x=0$ and $0 \leqslant t<t_{0}$ (where $|\gamma|>1$ ), and if the function $t \mapsto u_{t}(0, t)$ is not bounded as $t \stackrel{<}{\rightarrow} t_{0}$, the following estimates hold for some $C>0$ with $l=\min \left(1,2 \frac{\gamma+1}{\gamma-1}\right)$ if $0 \leqslant t<t_{0}$ :

$$
\begin{aligned}
& \left|\partial_{t}^{j} u(0, t)-\frac{4(-1)^{j}}{1-\gamma} \frac{1}{\left(t-t_{0}\right)^{j}}\right| \leqslant C\left(t_{0}-t\right)^{l-j} \quad \text { if } j=1,2, \\
& \left|u(0, t)-\frac{4}{\gamma-1} \ln \left(t_{0}-t\right)\right| \leqslant C, \\
& \left|\left(\frac{1}{2} u_{\eta}^{2}-u_{\eta \eta}\right)(0, t)-\frac{16 \gamma(\gamma+1)}{(\gamma-1)^{2}} \frac{1}{\left(t-t_{0}\right)^{2}}\right| \\
& \quad \leqslant C\left(t_{0}-t\right)^{l-2} .
\end{aligned}
$$

(II) If $F$ also satisfies (2.17) (with $g \in C^{1}(\mathbb{R})$ ), if $u=0$ when $x=0$ and $0 \leqslant t<t_{0}$, and if the function $t \mapsto u_{x}(0, t)$ is not bounded as $t \stackrel{<}{\rightarrow} t_{0}$, then the following estimates hold for some $C>0$ if $0 \leqslant t<t_{0}$ :

$$
\begin{aligned}
& \text { (1) }\left|\partial_{t}^{j} \partial_{x} u(0, t)-\frac{4}{\left(t_{0}-t\right)^{j+1}}\right| \leqslant \frac{C}{\left(t_{0}-t\right)^{j}}, \\
& \text { (2) }\left|\left(\frac{1}{2} u_{\eta}^{2}-u_{\eta \eta}\right)(0, t)-\frac{16}{\left(t-t_{0}\right)^{2}}\right| \leqslant \frac{C}{t_{0}-t} .
\end{aligned}
$$

(III) If $F$ also satisfies (2.10), if $u_{x}-u_{t}=0$ when $x=0$ and $0 \leqslant t<t_{0}$ and if the function $t \mapsto u_{t}(0, t)$ is not bounded as $t \stackrel{<}{\rightarrow} t_{0}$, the following estimates hold for some $C>0$ if $0 \leqslant t<t_{0}<t+\frac{1}{2 \mathrm{e}}$ :

$$
\begin{aligned}
& \text { (1) }\left|u(0, t)-\ln \frac{8}{\left(t-t_{0}\right)^{2}}+\ln \ln \frac{1}{\left(t-t_{0}\right)^{2}}\right| \leqslant C W(t), \\
& \text { (2) }\left|u_{t}(0, t)-\frac{2}{t_{0}-t}\right| \leqslant \frac{C}{t_{0}-t} W(t),
\end{aligned}
$$




$$
\text { (3) } \begin{aligned}
& \left|\left(\frac{1}{2} u_{\eta}^{2}-u_{\eta \eta}\right)(0, t)+\frac{4}{\left(t-t_{0}\right)^{2} \ln \left(t_{0}-t\right)}\right| \\
& \leqslant \frac{C}{\left(t_{0}-t\right)^{2}} \frac{W(t)}{\left|\ln \left(t_{0}-t\right)\right|},
\end{aligned}
$$

where $W(t)=\frac{\ln \left|\ln \left(t_{0}-t\right)\right|}{\left|\ln \left(t_{0}-t\right)\right|}$.

A crucial role will be played in this section by two simple conservation laws which we are going to derive now. Assume that $\mathcal{D}$ is an open subset of $\left(\overline{\mathbb{R}^{+}}\right)^{2}$ and that $u \in C^{3}(\mathcal{D})$. Let $g \in C^{1}(\mathbb{R})$ be such that $g^{\prime}=F-F^{\prime}$. Then it is readily verified that the following conservation laws hold:

$$
\begin{aligned}
& \partial_{\eta}\left(\frac{1}{2} u_{\xi}^{2}-u_{\xi \xi}\right)=\partial_{\xi}(g(u)), \\
& \partial_{\xi}\left(\frac{1}{2} u_{\eta}^{2}-u_{\eta \eta}\right)=\partial_{\eta}(g(u)) .
\end{aligned}
$$

In the proof of Propositions 5.1 and 5.2, we shall use (5.1); (5.2) will be used later on. Let us integrate (5.1) over the triangular domain $D_{T}$ with vertices $(0,0),(T, 0),(0, T), 0<T<t_{0}$. Put

$$
E(T)=\int_{0}^{T}\left(\frac{1}{2} u_{\xi}^{2}-u_{\xi \xi}-g(u)\right)(x, 0) d x, \quad \mathcal{F}=F+g .
$$

If $u_{x}+\gamma u_{t}=0$ on $\{0\} \times[0, T]$, we obtain

$$
\begin{aligned}
& \int_{0}^{T}\left(\frac{(1-\gamma)^{2}}{2} u_{t}^{2}-2(1-\gamma) u_{t t}+\mathcal{F}(u)\right)(0, t) d t \\
& \quad=2 \int_{0}^{T} g(u)(s, T-s) d s+E(T) .
\end{aligned}
$$

If $u=0$ on $\{0\} \times[0, T]$ instead, we obtain

$$
\int_{0}^{T}\left(\frac{1}{2} u_{x}^{2}-2 u_{t x}\right)(0, t) d t+\mathcal{F}(0)=2 \int_{0}^{T} g(u)(s, T-s) d s+E(T) .
$$

Proof of Proposition 5.1(I). - We shall first show that

$$
\text { the function } t \mapsto u_{t t}(0, t) \text { belongs to } L^{\infty}\left(\left[0, t_{0}[)\right.\right. \text {. }
$$


Applying $\partial_{T}$ to (5.3), we see that it is enough to show that the function

$$
T \mapsto \partial_{T}\left(\int_{0}^{T} g(u)(s, T-s) d s\right)
$$

belongs to $L^{\infty}\left(\left[0, t_{0}[)\right.\right.$, which in turn will be a consequence of the fact that

$$
\partial^{\alpha} u \in L^{\infty}(\mathcal{T}) \text { if }|\alpha|=1,
$$

where $\mathcal{T}$ is the triangular domain with vertices $(0,0),\left(\frac{t_{0}}{2}, \frac{t_{0}}{2}\right),\left(0, t_{0}\right)$. Let us check (5.6). If we multiply the equation $-u_{t t}+u_{x x}+F(u)=0$ by $u_{x}$, we obtain that

$$
\partial_{t}\left(-u_{t} u_{x}\right)+\partial_{x}\left(\frac{u_{t}^{2}+u_{x}^{2}}{2}+\mathcal{F}(u)\right)=0
$$

Integrating this last relation over the triangular domain $\tilde{\mathcal{T}}$ with vertices $(x, t),(0, t+x),(0, t-x)$ and using the divergence formula, we obtain that

$$
\left|\int_{0}^{x} \mathcal{F}(u)(s, t \pm(x-s)) d s\right| \leqslant C \text {. }
$$

Now

$$
u_{\xi}(x, t)=u_{\xi}(0, t+x)-\int_{0}^{x} F(u)(s, t+x-s) d s
$$

and

$$
u_{\eta}(x, t)=u_{\eta}(0, t-x)+\int_{0}^{x} F(u)(s, t-x+s) d s .
$$

Furthermore, it follows from (2.17) that $g(u) \geqslant-C$ in $\mathcal{T}$. This is clear if $\gamma>1$, and follows with the help of Lemma A.1 of Appendix A if $\gamma<1$. Hence $F(u) \leqslant \mathcal{F}(u)+C$, and (5.6) follows. Hence (5.5) holds. Now denote by $U$ the solution of the Cauchy problem $\square U=0$ in $\mathcal{T}$, $\partial_{x}^{j} U=\partial_{x}^{j} u$ when $x=0,0<t<t_{0}$, and $j=0,1$. Then of course

$$
u(x, t)=U(x, t)-\frac{1}{2} \iint_{\tilde{\mathcal{T}}} F(u)(y, s) d y d s,
$$


so it follows with the help of (5.6) that $\partial^{\alpha} u \in L^{\infty}(\mathcal{T})$ if $|\alpha| \leqslant 2$. Actually we have that

$$
\text { the function } t \mapsto u_{t t}(0, t) \text { belongs to } C\left(\left[0, t_{0}\right]\right) \text {. }
$$

Indeed, if $|\alpha|=1$, the function $t \mapsto \partial^{\alpha} u(0, t)$ belongs to $C\left(\left[0, t_{0}\right]\right)$ since the function $t \mapsto \partial_{t} \partial^{\alpha} u(0, t)$ belongs to $L^{\infty}\left(\left[0, t_{0}[)\right.\right.$. Now the function

$$
T \mapsto \partial_{T}\left(\int_{0}^{T} g(u)(s, T-s) d s\right)
$$

is Lipschitz continuous on $\left[0, t_{0}\left[\right.\right.$ since $\partial^{\alpha} u \in L^{\infty}(\mathcal{T})$ if $|\alpha| \leqslant 2$. Then (5.7) follows easily if we apply $\partial_{T}$ to (5.3). Using (5.7) and the relations $u_{x}+\gamma u_{t}=0$ if $x=0, u_{x x}=u_{t t}-F(u)$, we obtain that the function $t \mapsto \partial^{\alpha} u(0, t)$ belongs to $C\left(\left[0, t_{0}\right]\right)$ if $|\alpha| \leqslant 2$. Proposition 5.1(I) now follows from standard results corresponding to Theorem 2.1(1) for the Cauchy problem for the equation $\square u=F(u)$ in $\mathcal{T}$ with Cauchy data on $\{0\} \times\left[0, t_{0}\right]$.

Proof of Proposition 5.1(II). - Arguing as for (I), but with (5.3) replaced by (5.4) and Lemma A.1 of Appendix A replaced by Lemma 3.1 , we obtain again that the function $t \mapsto \partial^{\alpha} u(0, t)$ belongs to $C\left(\left[0, t_{0}\right]\right)$ if $|\alpha| \leqslant 2$; and we can then conclude as in (I).

Proof of Proposition 5.2(I). - Assume first that $\gamma>1$. Since $g \in$ $L^{\infty}(\mathbb{R})$, it follows from $(5.3)$ that

$$
\begin{aligned}
& \text { the function } t \mapsto 2(\gamma-1) u_{t}(0, t)+\int_{0}^{t}\left(\frac{(\gamma-1)^{2}}{2} u_{s}^{2}\right. \\
& +F(u))(0, s) d s \text { belongs to } L^{\infty}\left(\left[0, t_{0}[) .\right.\right.
\end{aligned}
$$

Since $F$ is bounded below, it follows from (5.8) that $u_{t}$ (and so $u$ ) are bounded above if $x=0$ and $0 \leqslant t<t_{0}$, so that $F(u)(0, t) \in L^{\infty}\left(\left[0, t_{0}[)\right.\right.$. Hence it follows from (5.8) that

$$
\text { the function } t \mapsto u_{t}(0, t)+\frac{\gamma-1}{4} \int_{0}^{t} u_{s}^{2}(0, s) d s
$$

belongs to $L^{\infty}\left(\left[0, t_{0}[)\right.\right.$. 
Since the function $t \mapsto u_{t}(0, t)$ does not belong to $L^{\infty}\left(\left[0, t_{0}[)\right.\right.$, it follows from (5.9) that one can find a sequence $\left(t_{k}\right)$ such that $t_{k} \nearrow t_{0}$ and $u_{t}\left(0, t_{k}\right) \searrow-\infty$. On the other hand it follows from (5.9) that $u_{t}\left(0, t_{2}^{\prime}\right) \leqslant$ $u_{t}\left(0, t_{1}^{\prime}\right)+C$ if $t_{1}^{\prime}<t_{2}^{\prime}<t_{0}$; hence $u_{t}(0, t) \rightarrow-\infty$ as $t \stackrel{<}{\rightarrow} t_{0}$. Put

$$
\zeta(t)=\left(\frac{\gamma-1}{4}\right)^{2} \int_{0}^{t} u_{s}^{2}(0, s) d s .
$$

It follows from (5.9) that $\sqrt{\zeta^{\prime}}-\zeta \in L^{\infty}\left(\left[0, t_{0}[)\right.\right.$. Since $\zeta^{\prime}(t) \rightarrow+\infty$ as $t \stackrel{<}{\rightarrow} t_{0}$, we therefore obtain that $\zeta(t) \rightarrow+\infty$ as $t \stackrel{<}{\rightarrow} t_{0}$ and so $\left(\zeta-C_{0}\right)^{2} \leqslant$ $\zeta^{\prime} \leqslant\left(\zeta+C_{0}\right)^{2}$ for some $C_{0}>0$ if $t$ is close to $t_{0}$. Hence

$$
\left|\zeta(t)-\frac{1}{t_{0}-t}\right| \leqslant C_{0}
$$

so using (5.9) we obtain that

$$
\left|u_{t}(0, t)-\frac{4}{\gamma-1} \frac{1}{t-t_{0}}\right| \leqslant C .
$$

Integrating (5.10) with respect to $t$ yields

$$
\left|u(0, t)-\frac{4}{\gamma-1} \ln \left(t_{0}-t\right)\right| \leqslant C .
$$

Differentiating (5.3) with respect to $T$ yields

$$
\begin{aligned}
& \left(\frac{(1-\gamma)^{2}}{2} u_{t}^{2}-2(1-\gamma) u_{t t}+\mathcal{F}(u)\right)(0, T) \\
& \quad=2 \int_{0}^{T} \partial_{T}(g(u)(s, T-s)) d s+2 g(u)(T, 0)+E^{\prime}(T) .
\end{aligned}
$$

Now we have that

$$
\left|\int_{0}^{T} \partial_{T}(g(u)(s, T-s)) d s\right| \leqslant \frac{C}{t_{0}-T} .
$$

Actually, it follows from (5.10), (5.11) that 


$$
\begin{aligned}
& \left|\int_{0}^{T}\left(\frac{u_{t}^{2}+u_{x}^{2}}{2}+\mathcal{F}(u)\right)(0, t) d t\right| \\
& \quad \leqslant \frac{8\left(1+\gamma^{2}\right)}{(\gamma-1)^{2}} \frac{1}{t_{0}-T}+C\left(\left|\ln \left(t_{0}-T\right)\right|+1\right) .
\end{aligned}
$$

Hence if we integrate the identity

$$
\partial_{t}\left(-u_{t} u_{x}\right)+\partial_{x}\left(\frac{u_{t}^{2}+u_{x}^{2}}{2}+\mathcal{F}(u)\right)=0
$$

over the triangular domain with vertices $(0,0),(T, 0),(0, T)$ and use the divergence formula, we easily obtain that

$$
\int_{0}^{T} F(u)(s, T-s) d s \leqslant \frac{8\left(1+\gamma^{2}\right)}{(\gamma-1)^{2}} \frac{1}{t_{0}-T}+C\left(\left|\ln \left(t_{0}-T\right)\right|+1\right) .
$$

Now $u_{\xi}(x, T-x)=u_{\xi}(T, 0)+\int_{x}^{T} F(u)(s, T-s) d s$ if $0 \leqslant x \leqslant T$, and $\int_{x}^{T} F(u)(s, T-s) d s \leqslant \int_{0}^{T} F(u)(s, T-s) d s+C$ since $F(u) \geqslant$ $-C_{0}$. Hence $u_{\xi}(x, T-x)$ is bounded above by the right-hand side of (5.15) (with a larger $C$ ). Therefore if we write $\partial_{t}(g(u))=\frac{1}{2} \partial_{\xi}(g(u))+$ $\frac{1}{2} \partial_{\eta}(g(u)),(5.13)$ follows easily. From (5.12), (5.10), (5.11), (5.13), we easily obtain that

$$
\left|u_{t t}(0, t)-\frac{4}{1-\gamma} \frac{1}{\left(t-t_{0}\right)^{2}}\right| \leqslant \frac{C}{t_{0}-t} .
$$

Since $u_{x}=-\gamma u_{t}$ when $x=0$ and $0 \leqslant t<t_{0}$, it follows then that $u_{\eta \eta}=2(1+\gamma) u_{t t}-F(u)$. A simple computation using (5.10), (5.11), (5.16) gives that

$$
\left|\left(\frac{1}{2} u_{\eta}^{2}-u_{\eta \eta}\right)(0, t)-\frac{16 \gamma(\gamma+1)}{(\gamma-1)^{2}} \frac{1}{\left(t-t_{0}\right)^{2}}\right| \leqslant \frac{C}{t_{0}-t} .
$$

This proves Proposition 5.2(I) if $\gamma>1$.

Assume now that $\gamma<-1$. Let $D_{t_{0}}$ be the triangular domain with vertices $(0,0),\left(t_{0}, 0\right),\left(0, t_{0}\right)$. Since $\gamma<-1$, it follows from Lemma A.1 of Appendix A that $u \geqslant-C$ in $D_{t_{0}}$. Since $g \in L^{\infty}\left(\mathbb{R}^{+}\right)$, it follows that (5.8) still holds, from which we obtain that 


$$
\begin{aligned}
u_{t}(0, t)= & \frac{1-\gamma}{4} \int_{0}^{t} u_{s}^{2}(0, s) d s \\
& +\frac{1}{2(1-\gamma)} \int_{0}^{t} F(u)(0, s) d s+G(t),
\end{aligned}
$$

where $G \in L^{\infty}\left(\left[0, t_{0}[) \cap C^{2}\left(\left[0, t_{0}[)\right.\right.\right.\right.$. Since the function $t \mapsto u_{t}(0, t)$ does not belong to $L^{\infty}\left(\left[0, t_{0}[)\right.\right.$, it follows from (5.18) that one can find a sequence $\left(t_{k}\right)$ such that $t_{k} \nearrow t_{0}$ and $u_{t}\left(0, t_{k}\right) \nearrow+\infty$. (5.18) shows that $u_{t}\left(0, t_{2}^{\prime}\right) \geqslant u_{t}\left(0, t_{1}^{\prime}\right)-C$ if $t_{1}^{\prime}<t_{2}^{\prime}<t_{0}$; therefore $u_{t}(0, t) \rightarrow+\infty$ as $t \stackrel{<}{\rightarrow} t_{0}$. Put $\hat{G}(t)=-\int_{t}^{t_{0}} G(s) d s, 0 \leqslant t<t_{0}, U(t)=u(0, t)-\hat{G}(t)$. From (5.18) it follows after differentiation with respect to $t$ that

$$
U^{\prime \prime}=\frac{1-\gamma}{4}\left(U^{\prime}+G\right)^{2}+\frac{1}{2(1-\gamma)} F(U+\hat{G}) .
$$

Now $U^{\prime}(t)>0$ for $t \in\left[t_{1}, t_{0}\right.$ [if $t_{1}$ is close to $t_{0}$. Put $m=\lim _{t \rightarrow t_{0}} U(t)$, so $\left.m \in] U\left(t_{1}\right),+\infty\right]$. Let $\left.\mathcal{U}:\right] U\left(t_{1}\right), m[\rightarrow] t_{1}, t_{0}[$ be the inverse function of $U$, and write $Z=U^{\prime} \circ \mathcal{U}, \mathcal{G}=G \circ \mathcal{U}, \hat{\mathcal{G}}=\hat{G} \circ \mathcal{U}$. From (5.19) it follows that

$$
\left(Z Z^{\prime}\right)(s)=\frac{1-\gamma}{4}(Z(s)+\mathcal{G}(s))^{2}+\frac{1}{2(1-\gamma)} F(s+\hat{\mathcal{G}}(s))
$$

if $s \in] U\left(t_{1}\right), m$ [, so if we put $\zeta(s)=Z^{2}(s)$, we obtain that

$$
\zeta^{\prime}(s)=\frac{1-\gamma}{2}(\sqrt{\zeta(s)}+\mathcal{G}(s))^{2}+\frac{1}{1-\gamma} F(s+\hat{\mathcal{G}}(s))
$$

if $s \in] U\left(t_{1}\right), m$ [. If $m<+\infty$, it follows from (5.21) that $\zeta^{\prime} \leqslant C(\zeta+1)$ for some $C>0$; hence $\zeta$ is bounded above as $s \stackrel{<}{\rightarrow} m$, which contradicts the fact that $u_{t}(0, t) \rightarrow+\infty$ as $t \stackrel{<}{\rightarrow} t_{0}$. Hence $m=+\infty$. Let us now check that

$$
\text { for some } B>0, \quad\left|\zeta(s)-B \mathrm{e}^{\frac{1-\gamma}{2} s}\right| \leqslant C \mathrm{e}^{\left(\frac{1-\gamma}{2}-\omega\right) s}
$$

if $\omega=-\max \left(\frac{\gamma-1}{4}, \frac{\gamma+1}{2}\right)$ and $s$ is large. If we integrate the identity $\frac{d}{d s}\left(\mathrm{e}^{-s}(F+g)(s)\right)=-\mathrm{e}^{-s} g(s)$ over $[t, \theta], t>0$, and let $\theta \rightarrow+\infty$, we obtain that $F(t)=\mathrm{e}^{t}+\psi(t)$, where $\psi(t)=-g(t)+\int_{t}^{\infty} \mathrm{e}^{t-s} g(s) d s$. If we put $M(s)=\frac{1}{1-\gamma} \mathrm{e}^{\hat{\mathcal{G}}(s)}, N(s)=\frac{1-\gamma}{2} \mathcal{G}^{2}(s)+\frac{1}{1-\gamma} \psi(s+\hat{\mathcal{G}}(s)), H(s)=$ 
$\mathrm{e}^{\frac{\gamma-1}{2} s} \zeta(s),(5.21)$ gives

$$
H^{\prime}=(1-\gamma) \mathcal{G} \mathrm{e}^{\frac{\gamma-1}{4} s} \sqrt{H}+M \mathrm{e}^{\frac{\gamma+1}{2} s}+N \mathrm{e}^{\frac{\gamma-1}{2} s}
$$

if $s>U\left(t_{1}\right)$, so that $\left|H^{\prime}\right| \leqslant C \mathrm{e}^{-\omega s} \sqrt{H+1}$ if $s>U\left(t_{1}\right)$, with $\omega$ as in (5.22). From this last bound on $\left|H^{\prime}\right|$, it readily follows, since $\omega>0$,

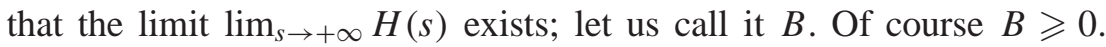
We are going to show that $B>0$. Since $\left|H^{\prime}(s)\right| \leqslant C \mathrm{e}^{-\omega s}$ for $s$ large, (5.22) will follow at once. For simplicity, put $a=\frac{1-\gamma}{4}, b=-\frac{1+\gamma}{2}$, so that $b=2 a-1>0$. Call $R(s)$ the right-hand side of (5.23). Define the sequence $\left(a_{k}\right)$ by $a_{1}=a, a_{k+1}=a+\frac{a_{k}}{2}$. Notice that $a_{k+1} \geqslant a_{k}+1$ if and only if $b \geqslant a_{k+1}$. Assume that $B=0$. Denote by $C_{j}, \tilde{C}_{j}$ various strictly positive constants. We are going to show by induction that

$$
b \geqslant a_{j} \text { and }|H(s)| \leqslant C_{j} \mathrm{e}^{-a_{j} s} \text { for } s \text { large, } \quad \text { if } j \in \mathbb{N} \backslash\{0\} .
$$

From (5.24) it will follow that $b \geqslant a+j-1$ for all $j \in \mathbb{N} \backslash\{0\}$. This contradiction of course will imply that $B>0$. Now if $b<a$, then $R(s)>0$ for $s$ large, which contradicts the fact that $B=0$. Hence $b \geqslant a$, so $|R(s)| \leqslant \tilde{C}_{1} \mathrm{e}^{-a s}$ for $s$ large, and therefore $|H(s)| \leqslant C_{1} \mathrm{e}^{-a s}$ for $s$ large since $B=0$. Hence (5.24) follows for $j=1$. Assume that (5.24) has been proved if $j \leqslant k$, and let us show that it still holds if $j=k+1$. Assume that $b<a_{k+1}$. Since $|H(s)| \leqslant C_{k} \mathrm{e}^{-a_{k} s}$ for $s$ large, it follows that $R(s)>0$ for $s$ large, which contradicts the fact that $B=0$. Hence $b \geqslant a_{k+1}$. But then $|R(s)| \leqslant \tilde{C}_{k+1} \mathrm{e}^{-a_{k+1} s}$ for $s$ large, so $|H(s)| \leqslant C_{k+1} \mathrm{e}^{-a_{k+1} s}$ for $s$ large since $B=0$. Hence (5.24) holds if $j=k+1$. Therefore we conclude that $B$ must be $>0$, and this completes the proof of (5.22).

Now (5.22) implies that $\left|\mathrm{e}^{\frac{\gamma-1}{4} s} Z(s)-\sqrt{B}\right| \leqslant C \mathrm{e}^{-\omega s}$ if $s>U\left(t_{1}\right)$, hence

$$
\left.\left|\mathrm{e}^{\frac{\gamma-1}{4} U(t)} U^{\prime}(t)-\sqrt{B}\right| \leqslant C \mathrm{e}^{-\omega U(t)} \quad \text { if } t \in\right] t_{1}, t_{0}[.
$$

Integrating (5.25), we obtain that

$$
\left.\left|\mathrm{e}^{\frac{\gamma-1}{4} U(t)}-\frac{1-\gamma}{4} \sqrt{B}\left(t_{0}-t\right)\right| \leqslant C \int_{t}^{t_{0}} \mathrm{e}^{-\omega U(s)} d s \quad \text { if } t \in\right] t_{1}, t_{0}[.
$$

This implies that

$$
\left|U(t)-\frac{4}{\gamma-1} \ln \left(t_{0}-t\right)\right| \leqslant C,
$$


so $\mathrm{e}^{-\omega U(t)} \leqslant C\left(t_{0}-t\right)^{l}$, with $l$ as in the statement of Proposition 5.2 and therefore (5.26) can be improved to

$$
\left|\mathrm{e}^{\frac{\gamma-1}{4} U(t)}-\frac{1-\gamma}{4} \sqrt{B}\left(t_{0}-t\right)\right| \leqslant C\left(t_{0}-t\right)^{1+l}
$$

which, together with (5.25), then implies that

$$
\left|u_{t}(0, t)-\frac{4}{1-\gamma} \frac{1}{t_{0}-t}\right| \leqslant C\left(t_{0}-t\right)^{-1+l} .
$$

Also (5.27) yields

$$
\left|u(0, t)-\frac{4}{\gamma-1} \ln \left(t_{0}-t\right)\right| \leqslant C .
$$

Let us check that (5.13) still holds. Actually, it follows from (5.28), (5.29) that (5.14) and (5.15) still hold (with $\left|\ln \left(t_{0}-T\right)\right|+1$ replaced by $\left(t_{0}-T\right)^{-1+l}$ in the right-hand side if $\left.-3<\gamma<-1\right)$. Reasoning as in the case where $\gamma>1$, we easily conclude that (5.13) still holds. Since it is clear that (5.12) also holds, it follows that

$$
\left|u_{t t}(0, t)-\frac{4}{1-\gamma} \frac{1}{\left(t-t_{0}\right)^{2}}\right| \leqslant C\left(t_{0}-t\right)^{l-2} .
$$

Arguing as for (5.17), we easily obtain that Proposition 5.2(I) (3) holds if $\gamma<-1$. This completes the proof of Proposition 5.2(I).

Proof of Proposition 5.2(II). - As before, let $D_{t_{0}}$ be the triangular domain with vertices $(0,0),\left(t_{0}, 0\right),\left(0, t_{0}\right)$. It follows from Lemma 3.1 that $u \geqslant-C$ in $D_{t_{0}}$, so that (5.4) implies that the function $t \mapsto u_{x}(0, t)-$ $\frac{1}{4} \int_{0}^{t} u_{x}^{2}(0, s) d s$ belongs to $L^{\infty}\left(\left[0, t_{0}[)\right.\right.$. If we then argue as for $(5.10)$ (with obvious modifications), we obtain that

$$
\left|u_{x}(0, t)-\frac{4}{t_{0}-t}\right| \leqslant C \text {. }
$$

(5.14) and (5.15) still hold with $\frac{1+\gamma^{2}}{(\gamma-1)^{2}}$ replaced by 1 in the right-hand side. It follows that (5.13) still holds, so if we differentiate (5.4) with respect to $T$, we obtain that

$$
\left|u_{t x}(0, t)-\frac{4}{\left(t-t_{0}\right)^{2}}\right| \leqslant \frac{C}{t_{0}-t} .
$$


Proposition 5.2(II) follows at once from (5.30) and (5.31).

Proof of Proposition 5.2(III). - Lemma A.2(3) of Appendix A shows that $u_{t}(0, t) \rightarrow+\infty$ as $t \stackrel{<}{\rightarrow} t_{0}$. (5.3) gives that

$$
u_{t}(0, t)=\frac{1}{2} \int_{0}^{t} u_{s}^{2}(0, s) d s+\frac{1}{4} \int_{0}^{t} F(u)(0, s) d s+G(t)
$$

if $0 \leqslant t<t_{0}$, where

$$
G(t)=u_{t}(0,0)-\frac{1}{4} E(t)+\frac{1}{4} \int_{0}^{t} g(u)(0, s) d s-\frac{1}{2} \int_{0}^{t} g(u)(s, t-s) d s .
$$

Using (2.11), Lemma A.2(2) of Appendix A, Lemma A.1 of Appendix A, we obtain that $|g(u)(y, s)| \leqslant C\left(t_{0}-y-s\right)^{-2 \alpha}$ if $y+s<t_{0}$, whence

$$
|G(t)| \leqslant C\left(t_{0}-t\right)^{-2 \alpha} \quad \text { if } 0 \leqslant t<t_{0} .
$$

Define $\hat{G}, U, \mathcal{U}, Z, \mathcal{G}, \hat{\mathcal{G}}, \psi, M, N, H$ as in Proposition 5.2(I), but with $\gamma$ replaced by $(-1)$. Notice that

$$
U^{\prime}(t)=u_{t}(0, t)-G(t)=\frac{1}{2} \int_{0}^{t} u_{s}^{2}(0, s) d s+\frac{1}{4} \int_{0}^{t} F(u)(0, s) d s,
$$

and that it follows from Lemma A.2(1) of Appendix A that $\int_{0}^{t} F(u)(0, s) d s \rightarrow+\infty$ as $t \stackrel{<}{\rightarrow} t_{0}$. Hence in particular there exists $t_{1} \in\left[0, t_{0}\right.$ [ such that $U^{\prime}(t)>0$ if $t>t_{1}$ and so $\mathcal{U}$ is well defined on ]$U\left(t_{1}\right),+\infty\left[\right.$ since $U^{\prime}(t) \rightarrow+\infty$ if $t \stackrel{<}{\rightarrow} t_{0}$. (5.23) can be written

$$
H^{\prime}=2 \mathcal{G} \mathrm{e}^{-s / 2} \sqrt{H}+P \text { if } s>U\left(t_{1}\right),
$$

where $P(s) \rightarrow \frac{1}{2}$ as $s \rightarrow+\infty$. By Lemma 4.1 of [3] and (5.32), it follows that $|\mathcal{G}(s)| \leqslant C \mathrm{e}^{2 \alpha s}$ if $s>U\left(t_{1}\right)$. If $\alpha>0$, let $k \in \mathbb{N} \backslash\{0\}$ be such that $(2 \alpha)^{k}<\frac{1}{2} \leqslant(2 \alpha)^{k-1}$. We are going to show that

$$
|\mathcal{G}(s)| \leqslant C \mathrm{e}^{(2 \alpha)^{j} s} \quad \text { if } s>U\left(t_{1}\right), j \in \mathbb{N} \text { and } j \leqslant k .
$$

Since we already know that (5.34) holds for $j \leqslant 1$, it is enough to show that if $|\mathcal{G}(s)| \leqslant C \mathrm{e}^{\beta s}$ for $s>U\left(t_{1}\right)$, where $\beta=(2 \alpha)^{j}$ for some $j \in \mathbb{N}$ 
with $j \leqslant k-1$, then $|\mathcal{G}(s)| \leqslant C \mathrm{e}^{2 \alpha \beta s}$ for $s>U\left(t_{1}\right)$. To achieve this, observe that (5.33) implies that $H^{\prime} \leqslant C \sqrt{H+1} \mathrm{e}^{\left(\beta-\frac{1}{2}\right) s}$ for $s>U\left(t_{1}\right)$, whence $H(s) \leqslant C \mathrm{e}^{(2 \beta-1) s}$ and therefore $U^{\prime}(\mathcal{U}(s)) \leqslant C \mathrm{e}^{\beta s}$, from which it follows that $\mathrm{e}^{\beta U(t)} \geqslant C\left(t_{0}-t\right)^{-1}$. By (5.32), it follows that $|\mathcal{G}(s)| \leqslant$ $C\left(t_{0}-\mathcal{U}(s)\right)^{-2 \alpha} \leqslant C \mathrm{e}^{2 \alpha \beta s}$ if $s>U\left(t_{1}\right)$, from which (5.34) follows. If $\alpha>0$, put $\tau=(2 \alpha)^{k}$ with $k$ as in (5.34); if $\alpha=0$, put $\tau=0$. So we have

$$
|\mathcal{G}(s)| \leqslant C_{1} \mathrm{e}^{\tau s} \quad \text { if } s>U\left(t_{1}\right)
$$

Let $K(s, H)=2 \mathcal{G}(s) \mathrm{e}^{-s / 2} \sqrt{H}+P(s)$ be the right-hand side of (5.33). Let us show that

$$
|K(s, H(s))| \leqslant C \quad \text { if } s \geqslant U\left(t_{1}\right) .
$$

To achieve this, put $\lambda=\frac{1}{2}-\tau$, where $\tau$ is as in (5.35), and define $\mathcal{E}=\left\{s \geqslant U\left(t_{1}\right), \sqrt{H(\sigma)} \mathrm{e}^{-\lambda \sigma} \leqslant C_{2}\right.$ if $\left.\sigma \in\left[U\left(t_{1}\right), s\right]\right\}$ where $C_{2}$ will be chosen later. If $C_{2}$ is large, then $\mathcal{E} \neq \emptyset$. $\mathcal{E}$ is closed, and let us show that $\mathcal{E}$ is open. Taking $C_{1}$ large enough, we may assume that $|P| \leqslant C_{1}$ so if $s \in \mathcal{E}$, it follows that $|K(\sigma, H(\sigma))| \leqslant 2 C_{1} C_{2}+C_{1}$ if $\sigma \in\left[U\left(t_{1}\right), s\right]$. But then (5.33) implies that $H(s) \leqslant\left(2 C_{1} C_{2}+C_{1}\right)\left(s-U\left(t_{1}\right)\right)+H\left(U\left(t_{1}\right)\right)$, which is $\leqslant \frac{C_{2}^{2}}{2} \mathrm{e}^{2 \lambda s}$ if $H\left(U\left(t_{1}\right)\right) \leqslant \frac{C_{2}^{2}}{2} \mathrm{e}^{2 \lambda U\left(t_{1}\right)}$ and $C_{2}$ is large enough. Then $s+\delta \in \mathcal{E}$ if $\delta>0$ is small, which shows that $\mathcal{E}$ is open. But then $\mathcal{E}=\left[U\left(t_{1}\right),+\infty[\right.$, from which (5.36) follows at once. Now (5.33) and (5.36) imply that $|H(s)| \leqslant C s$ if $s$ is large; together with (5.35), this implies that $K(s, H(s)) \rightarrow \frac{1}{2}$ as $s \rightarrow+\infty$. It follows that $\frac{H(s)}{s} \rightarrow \frac{1}{2}$ as $s \rightarrow+\infty$, so finally

$$
\mathrm{e}^{-\frac{1}{2} U(t)}(U(t))^{-1 / 2} U^{\prime}(t) \rightarrow 2^{-1 / 2} \quad \text { as } t \stackrel{<}{\rightarrow} t_{0} .
$$

Hence if we put $\Psi(s)=-\int_{s}^{\infty} \mathrm{e}^{-\sigma / 2} \sigma^{-1 / 2} d \sigma$, we obtain that

$$
U(t)=\Psi^{-1}\left(2^{-1 / 2}\left(t_{0}-t\right)(1+L(t))\right),
$$

where $L \in C^{3}\left(\left[0, t_{0}[)\right.\right.$ and $L(t) \longrightarrow_{t \rightarrow t_{0}}^{<} 0$. But we have the following estimate for $\Psi^{-1}$ :

$$
\left|\Psi^{-1}(\theta)-\ln \frac{4}{\theta^{2}}+\ln \ln \frac{1}{\theta^{2}}\right| \leqslant C \frac{\ln \ln \frac{1}{\theta^{2}}}{\ln \frac{1}{\theta^{2}}},
$$


if $\theta<0$ is close to 0 . Let us check (5.38). To achieve this, put first $f(s)=\mathrm{e}^{-s / 2} s^{-1 / 2}$. We shall first check that

$$
\left|f^{-1}\left(-\frac{\theta}{2}\right)-\ln \frac{4}{\theta^{2}}+\ln \ln \frac{1}{\theta^{2}}\right| \leqslant C \frac{\ln \ln \frac{1}{\theta^{2}}}{\ln \frac{1}{\theta^{2}}},
$$

if $\theta<0$ is close to 0 . Indeed, define $a:[1,+\infty[\rightarrow[1,+\infty[: s \mapsto s+\ln s$. Put $b=a^{-1}$. Then $b^{\prime}=\frac{b}{b+1}<1$ and $b(1)=1$. Hence $b(y)=y+R(y)$ with $R(y)<0$ if $y>1$. Actually we have for some $C_{1}, C_{2}>0$ :

$$
C_{1} \frac{\ln y}{y} \leqslant b(y)-y+\ln y \leqslant C_{2} \frac{\ln y}{y}
$$

if $y$ is large. Indeed it is easily checked that $\ln (1+z)>\alpha z$ if $\alpha>1$ and $\frac{1}{\alpha}-1<z<0$, whereas $\ln (1+z)<z$ then. Using this with $z=R(y) / y$ and the fact that $R(y)+\ln (y+R(y))=0$, we obtain (5.40), from which (5.39) follows easily. We can now prove (5.38). Integrating by parts, we obtain that $\Psi(s)=-2 \mathrm{e}^{-s / 2} s^{-1 / 2}(1+$ $J(s))$, where $J(s)=-\frac{1}{2} \int_{0}^{\infty} \mathrm{e}^{-\rho s / 2}(\rho+1)^{-3 / 2} d \rho$. Writing $] 0,+\infty[=$ ] $0,1[\cup[1,+\infty[$ and decomposing $J(s)$ accordingly, we readily obtain that $|J(s)| \leqslant C s^{-1}$. Since

$$
\Psi^{-1}(\theta)=f^{-1}\left(-\frac{\theta}{2\left(1+J\left(\Psi^{-1}(\theta)\right)\right)}\right),
$$

(5.38) follows from (5.39) after some simple computations. From (5.37) and (5.38) it follows that, if $t<t_{0}$ and $t$ is close to $t_{0}$,

$$
U(t)=\ln \frac{8}{\left(t-t_{0}\right)^{2}}-\ln \ln \frac{2}{\left(t-t_{0}\right)^{2}}+\mathcal{R}(t),
$$

with $\mathcal{R}(t) \rightarrow 0$ as $t \stackrel{<}{\rightarrow} t_{0}$. (5.41) implies in particular that $\left|t-t_{0}\right| \leqslant$ $C \mathrm{e}^{-U(t) / 2}(U(t))^{-1 / 2}$. On the other hand, it follows from (5.32) that $\left|M(s)-\frac{1}{2}\right| \leqslant C\left(t_{0}-\mathcal{U}(s)\right)^{1-2 \alpha}$, whence $\left|M(s)-\frac{1}{2}\right| \leqslant C \mathrm{e}^{\left(\alpha-\frac{1}{2}\right) s} s^{\alpha-\frac{1}{2}}$. Since $\tau \geqslant \alpha$ and since $|H(s)| \leqslant C s$ for large $s$, it follows from (5.33) that $\left|H^{\prime}(s)-\frac{1}{2}\right| \leqslant C \mathrm{e}^{-\lambda s} s^{1 / 2}$ when $s>U\left(t_{1}\right)$, where $\lambda=\frac{1}{2}-\tau$ as before. Hence $\left|H(s)-\frac{s}{2}\right| \leqslant C$ when $s>U\left(t_{1}\right)$. It follows that

$$
\left|\mathrm{e}^{-\frac{1}{2} U(t)}(U(t))^{-1 / 2} U^{\prime}(t)-2^{-1 / 2}\right| \leqslant C(U(t))^{-1},
$$

if $t<t_{0}$ and $t$ is close to $t_{0}$. Using (5.41) we may bound the right-hand side of (5.42) above by $C / \ln \frac{1}{t_{0}-t}$ if $t<t_{0}$ and $t$ is close to $t_{0}$. It follows 
in particular that $\left|\Psi(U(t))-2^{-1 / 2}\left(t-t_{0}\right)\right| \leqslant C\left(t_{0}-t\right) / \ln \frac{1}{t_{0}-t}$ if $t<t_{0}$ and $t$ is close to $t_{0}$, so from (5.38) we obtain that the function $\mathcal{R}$ of (5.41) satisfies

$$
|\mathcal{R}(t)| \leqslant C \frac{\ln \ln \frac{1}{t_{0}-t}}{\ln \frac{1}{t_{0}-t}},
$$

if $t<t_{0}$ and $t$ is close to $t_{0}$. The estimate (1) of Proposition 5.2(III) follows at once. Using (5.42), we easily find then that

$$
\left|U^{\prime}(t)-\frac{2}{t_{0}-t}\right| \leqslant \frac{C}{t_{0}-t} \frac{\ln \ln \frac{1}{t_{0}-t}}{\ln \frac{1}{t_{0}-t}},
$$

if $t<t_{0}$ and $t$ is close to $t_{0}$, and the estimate (2) of Proposition 5.2(III) follows immediately if we make use of (5.32).

Since $u_{\eta}(0, t)=0$ and $u_{\eta \eta}(0, t)=-F(u)(0, t)$, and since $\mid F(s)-$ $\mathrm{e}^{s} \mid \leqslant C \mathrm{e}^{\alpha s}$ for $s$ large, the estimate (3) of Proposition 5.2(III) follows from the estimate (1). The proof of Proposition 5.2 is complete.

\section{MORE ON SOLUTIONS AND PROOF OF THEOREMS 2.5 AND 2.8}

To prove Theorems 2.5 and 2.8 we shall need additional properties of solutions to $\square u=F(u)$ satisfying boundary conditions when $x=0$. Assume that $a>0$ and that $\chi:[0, a] \rightarrow \mathbb{R}^{+}$belongs to $C([0, a]) \cap$ $\left.\left.C^{1}(] 0, a\right]\right)$ and satisfies $\chi(0)=t_{0},-1<\chi^{\prime}(x) \leqslant 1$ if $\left.\left.x \in\right] 0, a\right]$. Put $\Lambda=\left\{(x, t) \in\left(\overline{\mathbb{R}^{+}}\right)^{2}, t<\chi(x), x+t \leqslant a+\chi(a)\right\}$. Let $F$ be as in (2.7), and assume that $u \in C^{3}(\Lambda)$ and that $\square u=F(u)$ in $\Lambda$. Also assume that either (1) $F$ also satisfies (2.17), $u_{x}+\gamma u_{t}=0$ when $x=0$ and $0 \leqslant t<t_{0}$, where $|\gamma|>1$, or (2) $F$ also satisfies (2.17), $u=0$ when $x=0$ and $0 \leqslant t<t_{0}$, else (3) $F$ also satisfies (2.10), $u_{x}-u_{t}=0$ when $x=0$ and $0 \leqslant t<t_{0}$. We shall need the next two propositions in order to prove Theorems 2.5 and 2.8 .

Proposition 6.1. - Assume that for all $x \in] 0, a]$, the following holds: $\left|\chi^{\prime}(x)\right|<1$ and $u(y, s) \rightarrow+\infty$ as $(y, s) \in \Lambda$ and $(y, s) \rightarrow$ $(x, \chi(x))$. Also assume that the function $t \mapsto u_{x}(0, t)$ does not belong to $L^{\infty}\left(\left[0, t_{0}[)\right.\right.$. Then $\chi^{\prime}(x) \rightarrow-1$ as $x \stackrel{>}{\rightarrow}$.

Proposition 6.2. - Assume that $\chi(x)=x+t_{0}$ for all $\left.\left.x \in\right] 0, a\right]$. Then the function $t \mapsto u_{x}(0, t)$ belongs to $L^{\infty}\left(\left[0, t_{0}[)\right.\right.$. 
We start with some preparations for the proof of Propositions 6.1 and 6.2. We shall put as before $\xi=\frac{x+t}{2}, \eta=\frac{t-x}{2}$, and also $X=\xi-\frac{t_{0}}{2}$, $Y=\eta-\frac{t_{0}}{2}$. Since $\chi^{\prime}(x)>-1$ if $x>0$, the curve $t=\chi(x), 0 \leqslant x \leqslant a$, is given by $Y=J(X), 0 \leqslant X \leqslant b$, where $b=\left(a+\chi(a)-t_{0}\right) / 2$. We may and shall assume that $a$ is so small that $J(X)>-\frac{t_{0}}{2}$ if $0 \leqslant X \leqslant b$. We shall have to consider the following two cases:

case (1) (the case of Proposition 6.1) $\left|\chi^{\prime}(x)\right|<1$ if $0<x \leqslant a$, so that $J(X)<0$ if $0<X \leqslant b$;

case (2) (the case of Proposition 6.2) $\chi(x)=x+t_{0}$ if $0<x \leqslant a$, so that $J(X)=0$ if $0<X \leqslant b$.

Define $\mathcal{D}=\left\{(X, Y) \in \mathbb{R}^{2}, 0 \leqslant X \leqslant b,-\frac{t_{0}}{2} \leqslant Y<J(X)\right\}$ in case (1) and $\mathcal{D}=\left\{(X, Y) \in \mathbb{R}^{2},-\frac{t_{0}}{2} \leqslant X \leqslant b,-\frac{t_{0}}{2} \leqslant Y<0\right\}$ in case (2).

In case (2), we shall denote by $u^{*}$ a $C^{3}$ extension of $u$ to $\{(x, t) \in$ $\left.\mathbb{R}^{2}, 0 \leqslant x+t \leqslant t_{0}+2 b, 0 \leqslant t<x+t_{0}\right\}$. If $\gamma<-1$ or if the Dirichlet condition is satisfied, we may and shall assume that $u^{*}$ is bounded; this is possible since Lemmas A.1 (of Appendix A) and 3.1 show that $u$ is bounded below if $0 \leqslant x \leqslant-t+t_{0}+2 b$ and $0 \leqslant t<x+t_{0}$. In case (1), we just put $u^{*}=u$. Define a function $K$ on $\mathcal{D}$ by the relation $K(X, Y)=\int_{0}^{x} g\left(u^{*}\right)(s, t-x+s) d s$. Put $Q(X)=X$ if $-\frac{t_{0}}{2} \leqslant X \leqslant 0$, $Q(X)=J(X)$ if $0 \leqslant X \leqslant b$ (so that $Q(X)=0$ if $0 \leqslant X \leqslant b$ in case (2)). Finally define the function $L$ on $\mathcal{D}$ by the relation $L(X, Y)=$ $-\int_{Y}^{Q(X)} K(X, \tilde{Y}) d \tilde{Y}$. Because of (5.2), one can find $H_{1} \in C^{2}(\Lambda)$ such that $\partial_{\xi} H_{1}=g(u)$ and $\partial_{\eta} H_{1}=\frac{1}{2} u_{\eta}^{2}-u_{\eta \eta}$ in $\Lambda$. Hence if $H_{2}(\xi, \eta)=$ $H_{1}(x, t)$ and $K_{2}(\xi, \eta)=K(X, Y)$, we obtain that $H_{2}(\xi, \eta)=H_{2}(\eta, \eta)+$ $K_{2}(\xi, \eta)$ if $(\xi-\eta, \xi+\eta) \in \Lambda$ and $\left(\xi-\frac{t_{0}}{2}, \eta-\frac{t_{0}}{2}\right) \in \mathcal{D}$. If we put $w=$ $\mathrm{e}^{-u / 2}$ in $\Lambda$, we have

$$
2 \frac{w_{\eta \eta}}{w}=\frac{1}{2} u_{\eta}^{2}-u_{\eta \eta}=\partial_{\eta} H_{1}
$$

Put $\tilde{w}(X, Y)=w(x, t), Z=\tilde{w} \mathrm{e}^{-L / 2}$. We obtain from (6.1) that

$$
\begin{aligned}
& \partial_{Y}^{2} Z+K \partial_{Y} Z+M Z=0 \\
& \quad \text { if }\left(X-Y, X+Y+t_{0}\right) \in \Lambda \text { and }(X, Y) \in \mathcal{D},
\end{aligned}
$$

where $M(X, Y)=-\frac{1}{2} \partial_{Y}\left(H_{2}\left(Y+\frac{t_{0}}{2}, Y+\frac{t_{0}}{2}\right)\right)+\frac{1}{4} K^{2}(X, Y)$. Notice that $\partial_{Y}\left(H_{2}\left(Y+\frac{t_{0}}{2}, Y+\frac{t_{0}}{2}\right)\right)=\left(g(u)+\frac{1}{2} u_{\eta}^{2}-u_{\eta \eta}\right)\left(0,2 Y+t_{0}\right)$.

After these preliminaries, we are going to prove Proposition 6.1. 
Proof of Proposition 6.1. - First we are going to check the following:

$$
K \in L^{\infty}(\mathcal{D}) .
$$

Indeed, if $F$ satisfies (2.17) (and $|\gamma|>1$ or the Dirichlet boundary condition is satisfied), we conclude (with the help of Lemma A.1 (of Appendix A) and of Lemma 3.1) that $g(u)$ is bounded if $0 \leqslant t<\chi(x)$ and $0 \leqslant x \leqslant t_{0}+2 b-t$, and (6.3) is then obvious. On the other hand, if $F$ satisfies (2.10) and $\gamma=-1$, we can write with the help of Lemma A.2 (2) of Appendix A that $\int_{0}^{x}|g(u)(s, t-x+s)| d s \leqslant C \int_{0}^{x} \Psi^{-2 \alpha} d s$ when $(x, t) \in \Lambda$, where

$$
\Psi=\chi(s)-s-t+x=(x-s)\left(1-\frac{\chi(x)-\chi(s)}{x-s}\right)+\chi(x)-t .
$$

Since, by Theorem $2.4, \frac{\chi(x)-\chi(s)}{x-s} \leqslant \theta<1$ if $0 \leqslant s<x$, it follows that $\Psi>(1-\theta)(x-s)$ if $0 \leqslant s<x$ and $(x, t) \in \Lambda$, and (6.3) follows easily.

Define $r(X, Y)=\mathrm{e}^{-L(X, Y)}$ if $(X, Y) \in \mathcal{D}$. It follows from (6.2) that $\partial_{Y}\left(r \partial_{Y} Z\right)+r M Z=0$ in $\mathcal{D}$. Using (6.3), we see that there exists $\omega>0$ such that $\frac{1}{r(X, Y)} \geqslant \omega$ if $(X, Y) \in \mathcal{D}$. Define $\mathcal{S}(X, Y)=\omega J(X)-$ $\int_{Y}^{J(X)} \frac{d \tilde{Y}}{r(X, \tilde{Y})}$ when $(X, Y) \in \mathcal{D}$. Notice for later use that $\mathcal{S}(X, Y) \leqslant \omega Y$ if $(X, Y) \in \mathcal{D}$; indeed $\partial_{Y} \mathcal{S} \geqslant \omega$ in $\mathcal{D}$ and $\mathcal{S}(X, J(X))=\omega J(X)$. Define $\tilde{\mathcal{D}}=\left\{(X, \mathcal{S}(X, Y)) \in \mathbb{R}^{2},(X, Y) \in \mathcal{D}\right\}$. Put $S=\mathcal{S}(X, Y), U(X, S)=$ $Z(X, Y)$. It follows easily from (6.2) that

$$
\partial_{S}^{2} U-q(X, S) U=0 \quad \text { if }(X, S) \in \tilde{\mathcal{D}},
$$

where $q(X, S)=-\left(r^{2} M\right)(X, Y)$. Notice that

$$
U(X, S) \rightarrow 0 \quad \text { if } 0<X \leqslant b \text { and } S \stackrel{<}{\rightarrow} \omega J(X) .
$$

Fix $\left.x_{0} \in\right] 0, a\left[\right.$. Since $\left|\chi^{\prime}(x)\right|<1$, it follows by standard arguments (already used in the proof of (6.1) of [3]) that, near $x_{0}, t=\chi(x)$ is the blow-up curve of $u$ considered as a solution of a Cauchy problem for the equation $\square u=F(u)$ with initial data on $\left\{\left(y, \chi\left(x_{0}\right)-\delta\right) \in\left(\mathbb{R}^{+}\right)^{2}, \mid y-\right.$ $x_{0} \mid \leqslant \delta+\varepsilon$, where $\delta>0$ is small and $\varepsilon>0$ is small with respect to $\delta$. Put, as in section 4 of [3], $u_{\lambda}(x, t)=u\left(x_{0}+\lambda x, \chi\left(x_{0}\right)+\lambda t\right)+2 \ln \lambda$, and define $V_{\tau}(x, t)=\ln \frac{2\left(1-\tau^{2}\right)}{(t-\tau x)^{2}}$, where $\tau=\chi^{\prime}\left(x_{0}\right)$. Now, one has in particular that $\partial^{\alpha} u_{\lambda}(1,-1) \rightarrow \partial^{\alpha} V_{\tau}(1,-1)$ if $|\alpha| \leqslant 2$ and $\lambda \stackrel{>}{\rightarrow} 0$. Actually this follows at once from the results of Sections 4,5,6 of [3], in particular 
from the analogue of (6.3) of [3] for $x_{0}>0$ (which corresponds to (7.4) of [1]). Hence

$$
\left(\mathrm{e}^{-\frac{1}{2} u_{\lambda}}\left(\partial_{t}-\partial_{x}\right) u_{\lambda}\right)(1,-1) \rightarrow\left(\mathrm{e}^{-\frac{1}{2} V_{\tau}}\left(\partial_{t}-\partial_{x}\right) V_{\tau}\right)(1,-1) \quad \text { as } \lambda \stackrel{>}{\rightarrow},
$$

that is

$$
-2 w_{\eta}\left(x_{0}+\lambda, \chi\left(x_{0}\right)-\lambda\right) \rightarrow\left(\frac{2\left(1+\chi^{\prime}\left(x_{0}\right)\right)}{1-\chi^{\prime}\left(x_{0}\right)}\right)^{1 / 2} \text { as } \lambda \stackrel{>}{\rightarrow} .
$$

Hence if we put, for $0<X \leqslant b$,

$$
\rho(X)=-\left(\frac{1+\chi^{\prime}(X-J(X))}{2\left(1-\chi^{\prime}(X-J(X))\right.}\right)^{1 / 2},
$$

it follows that $\partial_{Y} \tilde{w}(X, J(X)-\lambda) \rightarrow \rho(X)$ if $0<X \leqslant b$ and $\lambda \stackrel{>}{\rightarrow} 0$. Using this, it is not hard to check that

$$
\partial_{S} U(X, S) \rightarrow \rho(X) \quad \text { if } 0<X \leqslant b \text { and } S \stackrel{<}{\rightarrow} \omega J(X) .
$$

Let us complete the proof of Proposition 6.1 when either $|\gamma|>1$ or the Dirichlet condition is imposed. Using Proposition 5.2, we obtain that for some $C, m, \varepsilon>0, q(X, S) \geqslant \frac{C}{Y^{2}} \geqslant \frac{m}{S^{2}}$ if $(X, S) \in \tilde{\mathcal{D}}$ and $S \geqslant-\varepsilon$. We may and shall assume that $\varepsilon, b$ are so small that $\omega J(X)>-\varepsilon>-\frac{t_{0}}{2}$ if $0 \leqslant X \leqslant b$. Put $\tilde{\mathcal{D}}_{\varepsilon}=\{(X, S) \in \tilde{\mathcal{D}}, S>-\varepsilon\}$. Let $\zeta(X, S)$ be such that

$$
\begin{gathered}
\partial_{S}^{2} \zeta-\frac{m}{S^{2}} \zeta=0 \quad \text { if }(X, S) \in \tilde{\mathcal{D}}_{\varepsilon} \text { and } X>0 \\
\zeta(X, \omega J(X))=0 \quad \text { and } \quad \partial_{S} \zeta(X, \omega J(X))=\rho(X) \quad \text { if } 0<X \leqslant b .
\end{gathered}
$$

We are going to check that

$$
U(X, S) \geqslant \zeta(X, S) \quad \text { if }(X, S) \in \tilde{\mathcal{D}}_{\varepsilon} \text { and } X>0
$$

by adapting a standard comparison argument (used, e.g., in Theorem 9.2.1 of [5]). First we have if $(X, S) \in \tilde{\mathcal{D}}_{\varepsilon}$ and $X>0$ :

$$
\left(\zeta \partial_{S} U-U \partial_{S} \zeta\right)(X, S)=-\int_{S}^{\omega J(X)}(\zeta U)(X, \sigma)\left(q(X, \sigma)-\frac{m}{\sigma^{2}}\right) d \sigma
$$


because both sides have the same first derivative with respect to $S$, and are equal if $S=\omega J(X)$. Let us first check that $\left(\partial_{S} \zeta\right)(X, S)<0$ if $(X, S) \in \overline{\mathcal{D}}_{\varepsilon}$ and $X>0$. This is true if $S=\omega J(X)$, so fix $X$ and put $\mathcal{E}_{X}=\left\{S,(X, S) \in \overline{\mathcal{D}}_{\varepsilon},\left(\partial_{S} \zeta\right)(X, \sigma)<0\right.$ for all $\left.\sigma \in[S, \omega J(X)]\right\}$. Assume that there exists $S_{0}$ with $\left(X, S_{0}\right) \in \overline{\tilde{\mathcal{D}}}_{\varepsilon}$ and $S_{0} \notin \mathcal{E}_{X}$, and let $S^{*}$ be the largest $S \in\left[S_{0}, \omega J(X)\left[\right.\right.$ such that $\left(\partial_{S} \zeta\right)(X, S)=0$. Since $\zeta(X, J(X))=$ 0 , we have $\zeta\left(X, S^{*}\right)>0$. But (6.7) then shows that $\partial_{S}^{2} \zeta\left(X, S^{*}\right)>0$, so $S^{*}$ is a local minimum of the function $S \mapsto \zeta(X, S)$, which contradicts the fact that $\left(\partial_{S} \zeta\right)(X, S)<0$ if $\left.\left.S \in\right] S^{*}, J(X)\right]$. This contradiction shows that $\zeta(X, S)>0$ if $(X, S) \in \tilde{\mathcal{D}}_{\varepsilon}$ and $X>0$. Likewise $U(X, S)>0$ if $(X, S) \in \tilde{\mathcal{D}}_{\varepsilon}$ and $X>0$, so the right-hand side of (6.10) is $\leqslant 0$. Hence it follows from (6.10) that $\partial_{S}\left(\frac{U}{\zeta}\right) \leqslant 0$ if $(X, S) \in \tilde{\mathcal{D}}_{\varepsilon}$ and $X>0$. Since $\frac{U}{\zeta}(X, S) \rightarrow 1$ as $S \stackrel{<}{\rightarrow} \omega J(X),(6.9)$ follows at once.

Now (6.7), (6.8) can be solved explicitly. Put $\alpha=\frac{1}{2}(1+\sqrt{1+4 m})$, $\beta=\frac{1}{2}(1-\sqrt{1+4 m})$. Then $\left\{|S|^{\alpha},|S|^{\beta}\right\}$ is a fundamental system of solutions of (6.7) and a simple computation shows that

$$
\zeta(X, S)=\frac{\rho(X)}{\alpha-\beta}\left(\frac{|S|^{\beta}}{\omega^{\beta-1}|J(X)|^{\beta-1}}-\frac{|S|^{\alpha}}{\omega^{\alpha-1}|J(X)|^{\alpha-1}}\right) .
$$

Notice that $\alpha>1$ and that $\beta<0$. Assume that Proposition 6.1 is false, so that one can find $x_{k} \rightarrow 0$ with $\chi^{\prime}\left(x_{k}\right) \geqslant-1+c_{0}$ for some $c_{0}>0$ and all $k \in \mathbb{N} \backslash\{0\}$. Put $X_{k}=\left(x_{k}+\chi\left(x_{k}\right)-t_{0}\right) / 2$, so that $x_{k}=X_{k}-J\left(X_{k}\right)$. It is clear that there exists $\delta>0$ such that $\rho\left(X_{k}\right) \leqslant-\delta$ for all $k$. Now choose $S_{0}$ such that $-\varepsilon<S_{0}<\omega J(X)$ if $0 \leqslant X \leqslant b$. Let $\tilde{\mathcal{D}} \rightarrow \mathcal{D}:(X, S) \mapsto$ $(X, \mathcal{Y}(X, S))$ be the inverse diffeomorphism of $(X, Y) \mapsto(X, \mathcal{S}(X, Y))$ and put $Y_{k}=\mathcal{Y}\left(X_{k}, S_{0}\right)$. It is easily checked that one can find $\delta_{1}>0$ such that $Y_{k} \leqslant-\delta_{1}$ for all $k$. Passing to a subsequence if necessary, we may and shall assume that there exists $Y_{0} \in\left[-\frac{t_{0}}{2},-\delta_{1}\right]$ such that $Y_{k} \rightarrow Y_{0}$. By (6.11), we obtain that $\zeta\left(X_{k}, S_{0}\right) \rightarrow+\infty$, whence $U\left(X_{k}, S_{0}\right) \rightarrow+\infty$ by (6.9). It follows that $Z\left(X_{k}, Y_{k}\right) \rightarrow+\infty$, and so $\tilde{w}\left(X_{k}, Y_{k}\right) \rightarrow+\infty$. This contradiction proves Proposition 6.1 when either $|\gamma|>1$ or the Dirichlet condition is imposed.

Let us now complete the proof of Proposition 6.1 when $\gamma=-1$. Assuming as we may that $b$ is small and using Proposition 5.2, we see that one can find $C, m, \varepsilon>0$ such that $q(X, S) \geqslant C\left(Y^{2} \ln \frac{1}{|Y|}\right)^{-1} \geqslant$ $m\left(S^{2} \ln \frac{1}{|S|}\right)^{-1}$ and $|Y|<1,|S|<1$ if $(X, S) \in \tilde{\mathcal{D}}$ and $S \geqslant-\varepsilon$. Once more, we may and shall assume that $\varepsilon, b$ are so small that $\omega J(X)>-\varepsilon>$ $-\frac{t_{0}}{2}$ if $0 \leqslant X \leqslant b$, and put $\tilde{\mathcal{D}}_{\varepsilon}=\{(X, S) \in \tilde{\mathcal{D}}, S>-\varepsilon\}$. Let $\zeta(X, S)$ be 
such that

$$
\partial_{S}^{2} \zeta-m\left(S^{2} \ln \frac{1}{|S|}\right)^{-1} \zeta=0
$$

if $(X, S) \in \tilde{\mathcal{D}}_{\varepsilon}$ and $X>0$, and such that (6.8) holds. Then (6.9) still holds with the same proof. The following lemma will be used to provide something to replace $(6.11)$ in the present case.

LEMMA 6.1. - One can find a fundamental system $\left\{\zeta_{1}, \zeta_{2}\right\}$ of solutions of (6.12) such that, for $-\frac{1}{2} \leqslant S<0$,

$$
\begin{gathered}
\left|\zeta_{1}^{j /}(S)+S^{1-j}\left(\ln \frac{1}{|S|}\right)^{-m}\right| \leqslant C|S|^{1-j}\left(\ln \frac{1}{|S|}\right)^{-m-1}, \quad j=0,1, \\
\left|\zeta_{2}^{j /}(S)+(j-1)\left(\ln \frac{1}{|S|}\right)^{m}\right| \leqslant C|S|^{-j}\left(\ln \frac{1}{|S|}\right)^{m-1}, \quad j=0,1 .
\end{gathered}
$$

Lemma 6.1 is proved in Appendix B. Using Lemma 6.1 and taking (6.8) into account, we obtain that

$$
\begin{aligned}
\zeta(X, S)= & \rho(X)\left(\ln \frac{1}{|\omega J(X)|}\right)^{m} S\left(\ln \frac{1}{|S|}\right)^{-m}\left(1+R_{1}(X, S)\right) \\
& -\rho(X) \omega J(X)\left(\ln \frac{1}{|\omega J(X)|}\right)^{-m}\left(\ln \frac{1}{|S|}\right)^{m} \\
& \times\left(1+R_{2}(X, S)\right)
\end{aligned}
$$

where

$$
\left|R_{1}(X, S)\right|+\left|R_{2}(X, S)\right| \leqslant C\left(\left(\ln \frac{1}{|\omega J(X)|}\right)^{-1}+\left(\ln \frac{1}{|S|}\right)^{-1}\right)
$$

when $(X, S) \in \tilde{\mathcal{D}}_{\varepsilon}$ and $X>0, S \geqslant-\frac{1}{2}$.

We can now complete the proof of Proposition 6.1 when $\gamma=-1$. If this proposition was false, we could find $x_{k} \stackrel{>}{\rightarrow} 0$ with $\chi^{\prime}\left(x_{k}\right)>-1+c_{0}$ for some $c_{0}>0$ and all $k \in \mathbb{N} \backslash\{0\}$. Arguing as in the case $|\gamma|>1$ (or the Dirichlet case), but with (6.11) replaced by (6.13), we again reach a contradiction. The proof of Proposition 6.1 is complete.

Proof of Proposition 6.2. - Assume first that $\gamma=-1$. If the function $t \mapsto u_{t}(0, t)$ does not belong to $L^{\infty}\left(\left[0, t_{0}[)\right.\right.$, it follows (with the notations 
of Theorem 2.4) that $\varphi(0)=t_{0}$; indeed $\varphi(0) \geqslant t_{0}$, and if $\varphi(0)>t_{0}$, the function $t \mapsto u_{t}(0, t)$ belongs to $L^{\infty}\left(\left[0, t_{0}[)\right.\right.$. But if $\varphi(0)=t_{0}$, it follows from Theorem 2.4 that $\varphi(x) \leqslant \theta x+t_{0}$ for some $\left.\theta \in\right] 0,1[$ if $x \leqslant a$ and that $u(y, s) \rightarrow+\infty$ if $s<\varphi(y)$ and $(y, s) \rightarrow(x, \varphi(x))$. But this contradicts the fact that $\chi(x)=x+t_{0}$ in the definition of $\Lambda$. This contradiction proves Proposition 6.2 when $\gamma=-1$.

Assume now that $|\gamma|>1$ and that the function $t \mapsto u_{x}(0, t)$ does not belong to $L^{\infty}\left(\left[0, t_{0}[)\right.\right.$. Notice that (6.3) still holds in the present case; indeed (2.17) implies that the function $(x, t) \mapsto g\left(u^{*}\right)(x, t)$ is bounded if $\left(\frac{x+t-t_{0}}{2}, \frac{t-x-t_{0}}{2}\right) \in \mathcal{D}$.

We shall use the following result, which is proved in Appendix B.

LEMMA 6.2. - (6.2) has a fundamental system of solutions $\left\{Z_{1}(X, Y)\right.$, $\left.Z_{2}(X, Y)\right\}$ which belong to $C^{1}\left(\left\{(X, Y) \in \mathbb{R}^{2},-\frac{t_{0}}{2} \leqslant X \leqslant b,-\varepsilon \leqslant Y<\right.\right.$ 0\}) for some $\varepsilon>0$, and such that

$$
\begin{aligned}
& \left.\left.\left|\partial_{Y}^{j} Z_{1}(X, Y)+(-1)^{j+1}(1+\sigma)^{j}\right| Y\right|^{1+\sigma-j}|\leqslant C| Y\right|^{1+\sigma+l-j}, \\
& \quad j=0,1, \\
& \left.\left.\left|\partial_{Y}^{j} Z_{2}(X, Y)-\sigma^{j}\right| Y\right|^{-\sigma-j}|\leqslant C| Y\right|^{-\sigma+l-j}, \quad j=0,1,
\end{aligned}
$$

where $\sigma=\frac{\gamma+1}{\gamma-1}$ and $l=\min (1,2 \sigma)$.

Put as before $\tilde{w}(X, Y)=\mathrm{e}^{-u(x, t) / 2}$. It follows from Proposition 5.2 that

$$
\begin{aligned}
& \tilde{w}(X, X)=|X|^{1-\sigma} f_{1}(X) \quad \text { and } \\
& \partial_{Y} \tilde{w}(X, X)=\sigma|X|^{-\sigma} f_{1}(X)+f_{2}(X), \quad \text { if }-\frac{t_{0}}{2}<X<0,
\end{aligned}
$$

where, for some $C_{1}, C>0, C_{1} \leqslant f_{1}(X) \leqslant C$ and $\left|f_{2}(X)\right| \leqslant C|X|^{l-\sigma}$ if $-\frac{t_{0}}{2}<X<0$. Recall that $Z=\tilde{w} \mathrm{e}^{-L / 2}$, and $\partial_{Y}^{j} L(X, X)=0$ if $-\frac{t_{0}}{2}<X<$ 0 in particular if $j=0,1$. On the other hand, we may write $Z(X, Y)=$ $\sum_{1 \leqslant k \leqslant 2} A_{k}(X) Z_{k}(X, Y)$, and so $\sum_{1 \leqslant k \leqslant 2} A_{k}(X) \partial_{Y}^{j} Z_{k}(X, X)=\partial_{Y}^{j} \tilde{w}(X$, $X)$ if $j=0,1$. It then follows from (6.15) and from Lemma 6.2 that, in particular, $A_{2}(X)=-f_{1}(X) X+f_{3}(X)$, where $\left|f_{3}(X)\right| \leqslant C|X|^{1+l}$. Hence $A_{2}(0)=0$, and since $A_{2} \in C^{1}$ near 0 , we have $A_{2}^{\prime}(0)=$ $\lim _{X \rightarrow 0} \frac{A_{2}(X)}{X}$. Therefore $\lim _{X \rightarrow 0} f_{1}(X)$ exists; since $f_{1}(X) \geqslant C_{1}$, we also have $\lim _{X \rightarrow 0} f_{1}(X) \geqslant C_{1}$. Hence $A_{2}^{\prime}(0)<0$, and therefore $A_{2}\left(X_{0}\right)<0$ if $X_{0}>0$ is close to 0 . Since (6.3) still holds in the present situation, it 
follows that $\tilde{w}\left(X_{0}, Y\right) \rightarrow-\infty$ as $Y \stackrel{<}{\rightarrow} 0$, which is of course impossible. This contradiction proves Proposition 6.2 when $|\gamma|>1$.

Finally let us prove Proposition 6.2 when the boundary condition is the Dirichlet condition. The proof is identical with that of the case $|\gamma|>1$, with now $\sigma=1, l=1, f_{1}(X) \equiv 1$; actually (5.30) can be used to prove the analogue of (6.15) for $\partial_{Y} \tilde{w}(X, X)$ since Lemma 6.2 still holds with the same proof for these values of $\sigma, l$. The proof of Proposition 6.2 is complete.

We can now prove Theorem 2.5.

Proof of Theorem 2.5. - Assume that $\varphi\left(\overline{\mathbb{R}^{+}}\right) \subset \mathbb{R}^{+}$and put $t_{0}=\varphi(0)$. It follows from Theorem 2.4 that the function $t \mapsto u_{t}(0, t)$ does not belong to $L^{\infty}\left(\left[0, t_{0}[\right.\right.$ ). Proposition 6.1 (with $\chi=\varphi)$ then implies that $\varphi^{\prime}(x) \rightarrow-1$ as $x \stackrel{>}{\rightarrow} 0$. This implies Theorem 2.5 .

We now prove Theorem 2.8 with the help of Proposition 6.1. Until the end of this section, we shall suppose that the assumptions of Theorem 2.8 are satisfied.

If $(x, t) \in\left(\overline{\mathbb{R}^{+}}\right)^{2}$, let $K^{-}(x, t)=\left\{(y, s) \in\left(\overline{\mathbb{R}^{+}}\right)^{2}, s<t,|y-s|<t-s\right\}$ be the backward characteristic cone with vertex $(x, t)$, limited to $\left(\overline{\mathbb{R}^{+}}\right)^{2}$ (cf. Section 3). Recall that if $\mathcal{U}$ is an open subset of $\left(\overline{\mathbb{R}^{+}}\right)^{2}$, one says that $\mathcal{U}$ is an influence domain if $(x, t) \in \mathcal{U}$ implies that $\overline{K^{-}(x, t)} \subset \mathcal{U}$. The union $\mathcal{U}^{*}$ of all influence domains where a unique $C^{3}$ solution of (2.1), (2.2) (or (2.12)), (2.3) exists is the largest influence domain with such a property. If $x \geqslant 0$, one can find $t>0$ such that $\{x\} \times[0, t] \subset \mathcal{U}^{*}$. Put $\psi(x)=\sup \left\{t \geqslant 0,\{x\} \times[0, t] \subset \mathcal{U}^{*}\right\}$. If $\psi \not \equiv+\infty$, then $\psi$ is always $<+\infty$ and $\left|\psi\left(x_{1}\right)-\psi\left(x_{2}\right)\right| \leqslant\left|x_{1}-x_{2}\right|$ for all $x_{1}, x_{2} \geqslant 0$; in that case we shall put $\Sigma=\left\{(x, t) \in\left(\overline{\mathbb{R}^{+}}\right)^{2}, t=\psi(x)\right\}$. Actually, with the notations introduced in Section 2, we have $\mathcal{U}^{*}=\Omega, \psi=\varphi$, as will follow from Proposition 6.3 under the assumptions of Theorem 2.8 (and the same follows from Theorems 2.3 and 2.4 if $-1 \leqslant \gamma<1$ ). We shall prove the following result, which will help us to show that Proposition 6.1 is applicable with $\chi=\psi$.

Proposition 6.3. - Let the assumptions on $F, \psi_{0}, \psi_{1}$ and the boundary condition be as in Theorem 2.8. Assume that $\psi \neq \equiv+\infty$. If $x_{0}>0$, there exists an open neighborhood $U$ of $x_{0}$ in $\mathbb{R}^{+}$such that $\psi \in C^{1}(U)$ and $\left|\psi^{\prime}\right|<1$ in $U$. Moreover, if $x \in U, u(y, s) \rightarrow+\infty$ if $s<\psi(y)$ and $(y, s) \rightarrow(x, \psi(x))$. 
Proof of Proposition 6.3. - If $P=\left(x_{1}, t_{1}\right) \in\left(\overline{\mathbb{R}^{+}}\right)^{2}$, define $C_{l}(P)$, $C_{r}(P)$ as in Section 6 of [3], namely write $C_{l}(P)=\left\{(x, t) \in\left(\overline{\mathbb{R}^{+}}\right)^{2}\right.$, $\left.t<t_{1}, x-t=x_{1}-t_{1}\right\}, C_{r}(P)=\left\{(x, t) \in\left(\overline{\mathbb{R}^{+}}\right)^{2}, t<t_{1}, x+t=x_{1}+t_{1}\right\}$ for the backward half characteristics with positive and negative slope through $P$. Notice that if $P_{0}=\left(x_{0}, \psi\left(x_{0}\right)\right) \in \Sigma$ and if $P=(x, t) \in$ $C_{*}\left(P_{0}\right) \cap \Sigma$, where $*=l$ or $r$, then the closed interval with end points $P$ and $P_{0}$ is contained in $\Sigma$.

First case. Assume that $C_{*}\left(P_{0}\right) \cap \Sigma=\emptyset$ if $*=l$ and also if $*=r$. Then the following holds:

one can find an open neighborhood $U$ of $x_{0}$ in $\mathbb{R}^{+}$such that

$$
\begin{aligned}
& \psi \in C^{1}(U) \text { and }\left|\psi^{\prime}\right|<1 \text { in } U . \text { Moreover, if } \\
& x \in U, u(y, s) \rightarrow+\infty \text { if } s<\psi(y) \\
& \text { and }(y, s) \rightarrow(x, \psi(x)) .
\end{aligned}
$$

Actually the proof of (6.16) is the same as that of (6.1) of [3], so we may omit the details. Hence Proposition 6.3 holds if the first case occurs.

Second case. Assume that one can find $P_{1} \in C_{r}\left(P_{0}\right) \cap \Sigma$. Put $E^{+}=\left\{x>x_{0},\left(x, \psi\left(x_{0}\right)-\left(x-x_{0}\right)\right) \in \Sigma\right\} . E^{+} \neq \emptyset$. Put also $x^{*}=$ $\sup E^{+}, P^{*}=\left(x^{*}, \psi\left(x_{0}\right)-\left(x^{*}-x_{0}\right)\right)$. Then $P^{*} \in \Sigma$, so $t\left(P^{*}\right)>0$ and $\psi(x)=\psi\left(x_{0}\right)-\left(x-x_{0}\right)$ if $x_{0} \leqslant x \leqslant x^{*}$. It is clear that $C_{l}\left(P^{*}\right) \cap \Sigma=$ $\emptyset=C_{r}\left(P^{*}\right) \cap \Sigma$, so by the first case, $\psi \in C^{1}$ close to $x^{*}$ and $\left|\psi^{\prime}\right|<1$ close to $x^{*}$. This contradiction shows that the second case is impossible.

Third case. Assume that one can find $P_{2} \in C_{l}\left(P_{0}\right) \cap \Sigma$. Put $E^{-}=$ $\left\{x<x_{0},\left(x, \psi\left(x_{0}\right)+\left(x-x_{0}\right)\right) \in \Sigma\right\} . E^{-} \neq \emptyset$. Put $x^{* *}=\inf E^{-}, P^{* *}=$ $\left(x^{* *}, \psi\left(x_{0}\right)+\left(x^{* *}-x_{0}\right)\right)$. If $x^{* *}>0$, one can repeat the reasoning of the second case (with $C_{r}$ replaced by $C_{l}$ ) to conclude that this situation cannot happen. So we must have $x^{* *}=0$. Let us put $t_{0}=\psi\left(x_{0}\right)-x_{0}$, so that $\psi(x)=t_{0}+x$ if $x \leqslant x_{0}$. Proposition 6.2 shows that $t \mapsto u_{x}(0, t)$ belongs to $L^{\infty}\left(\left[0, t_{0}[)\right.\right.$. Then it follows from Proposition 5.1 that $u \in$ $C^{2}\left(\overline{D_{t_{0}}}\right)$. If we put as before $\tilde{w}(X, Y)=\mathrm{e}^{-u(x, t) / 2}, Z=\tilde{w} \mathrm{e}^{-L / 2}$, we are going to check that

$$
\text { for some } \delta, \varepsilon>0, Z(X, Y) \geqslant \delta \text { if } 0 \leqslant X \leqslant \varepsilon,-\frac{t_{0}}{2} \leqslant Y<0 \text {. }
$$

Since $u \in C\left(\overline{D_{t_{0}}}\right)$, it is clear that (6.17) holds if $X=0$. To prove (6.17), it is therefore sufficient to show that $Z_{X} \in L^{\infty}\left([0, b] \times\left[-\frac{t_{0}}{2}, 0[)\right.\right.$. To achieve 
this, put

$$
y=\left(\begin{array}{c}
Z_{Y} \\
Z
\end{array}\right), \quad \mathcal{A}=\left(\begin{array}{cc}
-K & -M \\
1 & 0
\end{array}\right)
$$

with $K, M$ as in (6.2). Using (6.2), we see that

$$
y_{Y}=\mathcal{A}(X, Y) y \quad \text { if } 0 \leqslant X \leqslant b,-\frac{t_{0}}{2} \leqslant Y<0
$$

while

$$
y\left(X,-\frac{t_{0}}{2}\right)=y_{0}(X) \quad \text { if } 0 \leqslant X \leqslant b,
$$

where $y_{0} \in C^{2}([0, b])$. Using the fact that $u \in C^{2}\left(\overline{D_{t_{0}}}\right)$, we obtain in particular that $\mathcal{A}, \mathcal{A}_{X} \in L^{\infty}\left([0, b] \times\left[-\frac{t_{0}}{2}, 0[)\right.\right.$. But then, standard estimates (see, e.g., Lemma 4.1, p. 54 of [4]) show that $y \in L^{\infty}([0, b] \times$ $\left[-\frac{t_{0}}{2}, 0[)\right.$, and differentiation of (6.18) with respect to $X$ shows that $y_{X} \in$ $L^{\infty}\left([0, b] \times\left[-\frac{t_{0}}{2}, 0[)\right.\right.$. Hence $Z_{X} \in L^{\infty}\left([0, b] \times\left[-\frac{t_{0}}{2}, 0[)\right.\right.$, and (6.17) follows. From (6.17) and from the fact that $u \in C\left(\overline{D_{t_{0}}}\right)$, it follows that $u$ is bounded above when $x+t<t_{0}+2 \varepsilon$ and $t<x+t_{0}$. Now $u_{t}+u_{x}$ is bounded if $0 \leqslant x \leqslant x_{0}+\psi\left(x_{0}\right)$ and $t=0$. Since $\square u=F(u) \geqslant-C_{0}$ if $x+t<x_{0}+\psi\left(x_{0}\right)$ and $t<x+t_{0}$, it is therefore clear that $u_{t}+u_{x}$ is bounded below if $x+t<x_{0}+\psi\left(x_{0}\right)$ and $t<x+t_{0}$. But then we conclude that $u$ is bounded below when $x+t<x_{0}+\psi\left(x_{0}\right)$ and $t<x+t_{0}$, since we know that $u$ is bounded if $x=0$ and $0 \leqslant t<t_{0}$. But then $u$ is bounded when $x+t<t_{0}+2 \varepsilon$ and $t<x+t_{0}$ if $\varepsilon<x_{0}$, and an application of Theorem A.1 of Appendix A shows that $u$ can be extended as a solution of (2.1), (2.2) (or $(2.1),(2.12)$ ) to a neighborhood of $\left(0, t_{0}\right)$ in $\left(\overline{\mathbb{R}^{+}}\right)^{2}$, which contradicts the fact that $P^{* *} \in \partial \mathcal{U}^{*}$. This contradiction shows that the third case is impossible. Summing up, only the first case is possible, and this proves Proposition 6.3.

We can now prove Theorem 2.8.

Proof of Theorem 2.8. - Proposition 6.3 immediately implies Theorem 2.8 when $x>0$ if we take $\varphi=\psi$. Now assume first that the function $t \mapsto u_{x}(0, t)$ does not belong to $L^{\infty}\left(\left[0, t_{0}[)\right.\right.$. If we take $\varphi=\chi$, Theorem 2.8 then follows at once from Proposition 6.1. (If $\gamma<-1$, $u(0, t) \rightarrow+\infty$ as $t \stackrel{<}{\rightarrow} t_{0}$ and $u_{\xi}$ is bounded below, so $u(x, t) \rightarrow+\infty$ if $(x, t) \rightarrow(0, \varphi(0)))$. If now the function $t \mapsto u_{x}(0, t)$ belongs to $L^{\infty}\left(\left[0, t_{0}[)\right.\right.$, we can repeat some arguments of the proof of Proposition 6.3. It follows from Proposition 5.1 that $u \in C^{2}\left(\overline{D_{t_{0}}}\right)$. Putting, as 
before, $\tilde{w}(X, Y)=\mathrm{e}^{-u(x, t) / 2}, Z=\tilde{w} \mathrm{e}^{-L / 2}$ and using arguments similar to those which lead to (6.17), we easily conclude that for some $\delta, \varepsilon>0, Z(X, Y) \geqslant \delta$ if $0 \leqslant X \leqslant \varepsilon$ and $-\frac{t_{0}}{2} \leqslant Y<J(X)$, where as before $Y=J(X)$ corresponds to $t=\varphi(x)$. This contradicts the fact that $u(y, s) \rightarrow+\infty$ as $(y, s) \rightarrow(x, \varphi(x))$, if $x>0$ is small. Hence the function $t \mapsto u_{x}(0, t)$ cannot belong to $L^{\infty}\left(\left[0, t_{0}[)\right.\right.$, and we have already seen in the beginning of this proof that Theorem 2.8 then follows.

\section{APPENDIX A}

We first collect a number of useful results from [3].

Lemma A.1 (Lemma 3.1. of [3]). - Let $D_{R}, \mathcal{U}$ be as in Section 3. Assume that $w \in C^{2}\left(\mathcal{U} \cap \overline{D_{R}}\right), F \in C\left(\mathcal{U} \cap \overline{D_{R}}\right)$, and that the following holds: $\square w=F$ in $\mathcal{U} \cap D_{R}, w_{x}+\gamma w_{t}=0$ if $x=0$ and $0<t<$ $\min (\psi(0), R)$, where $\gamma \in]-\infty, 1\left[\right.$, and $w=\psi_{0}, w_{t}=\psi_{1}$ if $0<x<R$ and $t=0$. Then the following holds: if $C_{0}>0$ and $F \geqslant-C_{0}$, one can find $C>0$ (depending on $\psi_{0}, \psi_{1}, R, C_{0}$, but not on $F$ ) such that $w \geqslant-C$ in $\mathcal{U} \cap \overline{D_{R}}$.

The following estimates have also been used (cf. [3]).

LEMMA A.2. - Assume that $F \in C^{1}(\mathbb{R})$ satisfies (2.7)(1) and (2.7)(2), that $\gamma=-1$, that $\psi_{j} \in C^{2-j}\left(\overline{\mathbb{R}^{+}}\right), j=0,1$, and that (2.4), (2.5) hold. Then there exist:

(a) $\left.\left.\varphi: \overline{\mathbb{R}^{+}} \rightarrow\right] 0,+\infty\right]$ such that $\varphi\left(\overline{\mathbb{R}^{+}}\right) \subset \mathbb{R}^{+}$or $\varphi\left(\overline{\mathbb{R}^{+}}\right)=\{+\infty\}$;

(b) $u \in C^{2}(\Omega)$, where $\Omega=\left\{(x, t) \in\left(\overline{\mathbb{R}^{+}}\right)^{2}, t<\varphi(x)\right\}$ such that $u$ is a solution of (2.1), (2.2), (2.3) in $\Omega$.

If $\varphi\left(\overline{\mathbb{R}^{+}}\right) \subset \mathbb{R}^{+}$, denote by $d(x, t)$ the distance from $(x, t)$ to the graph of $\varphi$. For any $R>0$, there exist $C, \delta>0$ such that:

(1) $\quad d(x, t) \mathrm{e}^{u(x, t)} \geqslant C^{-1}$ if $(x, t) \in \Omega \cap \overline{D_{R}}$;

(2) $\quad d^{2}(x, t) \mathrm{e}^{u(x, t)} \leqslant C$ if $(x, t) \in \Omega \cap \overline{D_{R}}$;

(3) $\quad u_{t}(x, t) \geqslant C^{-1} \ln \frac{1}{d(x, t)} \quad$ if $(x, t) \in \Omega \cap \overline{D_{R}}$ and $d(x, t) \leqslant \delta$.

Proof of Lemma A.2. - (1) can be proved as Lemma 4.1 of [3], and (2) as Lemma 4.2 of [3]. As for (3), it can be proved as Lemma 4.9 of [3], but with Lemma 4.7 of [3] replaced by (1). We may omit the details.

Assume that $t_{0}>0$ and $\beta>0$. If $\left.\varepsilon \in\right] 0, t_{0}\left[\right.$, put $D^{\varepsilon}=\{(x, t) \in$ $\left.\left(\mathbb{R}^{+}\right)^{2}, x>0,0<t<t_{0}-\varepsilon, x+t<t_{0}+\beta\right\}, D=\bigcup_{0<\varepsilon<\varepsilon_{0}} D^{\varepsilon}$. In the proof of Proposition 6.3, we have used the following standard result. 
THEOREM A.1. - (I) Assume that $F \in C^{2}(\mathbb{R})$ and let

$$
u \in \bigcap_{0<\varepsilon<\varepsilon_{0}} C^{3}\left(\overline{D^{\varepsilon}}\right)
$$

satisfy the following conditions:

$$
\begin{gathered}
\square u=F(u) \quad \text { if }(x, t) \in D, \\
u_{x}+\gamma u_{t}=0 \quad \text { if } x=0 \text { and } 0<t<t_{0},
\end{gathered}
$$

where $\gamma \neq 1$. If $u \in L^{\infty}(D)$, one can find an open neighborhood $V$ of $\left(0, t_{0}\right)$ in $\left(\overline{\mathbb{R}^{+}}\right)^{2}$ and $\tilde{u} \in C^{3}(V)$ with

$$
\begin{gathered}
\square \tilde{u}=F(\tilde{u}) \quad \text { if }(x, t) \in V, \\
\tilde{u}_{x}+\gamma \tilde{u}_{t}=0 \quad \text { if } x=0 \text { and }(0, t) \in V,
\end{gathered}
$$

such that $\tilde{u}=u$ in $V \cap D$.

(II) The same result holds if (A.2) is replaced by

$$
u=0 \text { if } x=0 \text { and } 0<t<t_{0},
$$

and (A.4) by

$$
\tilde{u}=0 \quad \text { if } x=0 \text { and }(0, t) \in V .
$$

Proof of Theorem A.1. - Representing $u$ in $D$ by formulas of type (3.4), (3.5), we easily conclude that $u \in C^{3}(\bar{D})$. It then suffices to find an open neighborhood $W$ of $\left(0, t_{0}\right)$ in $\left\{(x, t) \in\left(\overline{\mathbb{R}^{+}}\right)^{2}, t \geqslant t_{0}\right\}$ and $u^{*} \in C^{3}(W)$ such that $\square u^{*}=F\left(u^{*}\right)$ if $(x, t) \in W, \partial_{t}^{j} u^{*}=\partial_{t}^{j} u$ if $j=0,1,(x, t) \in W$ and $t=t_{0}$, and such that $u_{x}^{*}+\gamma u_{t}^{*}=0$ if $x=0$ and $(0, t) \in W$ in case (A.2) holds, whereas $u^{*}=0$ if $x=0$ and $(0, t) \in W$ in case (A.5) holds. But the existence of $W$ and $u^{*}$ is standard and follows, e.g., by the arguments of the proof of Theorem 2.1 of [3]. We may omit the details.

\section{APPENDIX B}

Proof of Lemma 6.1. - We shall use ideas and results of Chapter XI of [4]. Assume that $\zeta(S)$ satisfies (6.12) when $-\frac{1}{2} \leqslant S<0$. Put $-S=$ $t^{-1}$ (hence $\left.t \geqslant 2\right), \zeta(S)=t^{-1 / 2} v(\ln t), P(s)=\frac{1}{4}+\frac{m}{s}$. Then $v^{\prime \prime}(s)+$ 
$P(s) v(s)=0$ if $s \geqslant \ln 2$. Let us use a so-called Liouville transformation: take a new variable $\sigma$ such that $\frac{d \sigma}{d s}=P^{1 / 2}(s)$ and $\sigma(\ln 2)=1$, and write $z(\sigma)=P^{1 / 4}(s) v(s)$. Then

$$
z^{\prime \prime}(\sigma)-(1+\mathcal{B}(\sigma)) z(\sigma)=0 \quad \text { if } \sigma \geqslant 1,
$$

where $\mathcal{B} \in C^{\infty}\left(\left[1,+\infty[)\right.\right.$ and $|\mathcal{B}(\sigma)| \leqslant \frac{C}{\sigma^{3}}$. Now (B.1) has a fundamental system of solutions $\left\{z_{1}, z_{2}\right\}$ such that

$$
\begin{aligned}
& \left|z_{1}^{j /}(\sigma)-(-1)^{j} \mathrm{e}^{-\sigma}\right| \leqslant C \frac{\mathrm{e}^{-\sigma}}{\sigma^{2}}, \\
& \left|z_{2}^{j /}(\sigma)-\mathrm{e}^{\sigma}\right| \leqslant C \frac{\mathrm{e}^{\sigma}}{\sigma^{2}},
\end{aligned}
$$

if $\sigma \geqslant 1$ and $j=0,1$. Actually (B.2) follows from Corollary 9.2 of Chapter XI of [4] except for the fact that the right-hand sides of (B.2) are not given there. For the sake of completeness, we very briefly give some details. Put $v(\sigma)=\mathrm{e}^{\sigma} z(\sigma), w(\sigma)=\mathrm{e}^{-2 \sigma} v^{\prime}(\sigma)$, and assume in the rest of the proof that $\sigma \geqslant 1$. Then we obtain with the help of (B.1) that

$$
v^{\prime}(\sigma)=\mathrm{e}^{2 \sigma} w(\sigma), \quad w^{\prime}(\sigma)=\mathrm{e}^{-2 \sigma} \mathcal{B}(\sigma) v(\sigma) .
$$

(B.3) can be reduced to the system of integral equations

$$
\begin{aligned}
v(\sigma)= & \int_{T}^{\sigma} \mathrm{e}^{2 s} w(s) d s+v(T), \\
w(\sigma)= & \int_{T}^{\sigma} \mathrm{e}^{-2 s} \mathcal{B}(s)\left(\int_{T}^{s} \mathrm{e}^{2 r} w(r) d r\right) d s \\
& +v(T) \int_{T}^{\sigma} \mathrm{e}^{-2 s} \mathcal{B}(s) d s+w(T) .
\end{aligned}
$$

Lemma 9.1 of Chapter XI of [4] shows that $w(\sigma)$ has a finite limit $w(+\infty)$ as $\sigma \rightarrow+\infty$. We impose the conditions $v(T)=0, w(T)=$ 1, where $T$ is large; then it follows from the proof of Lemma 9.1 of Chapter XI of [4] that $w(+\infty) \neq 0$. Put $v_{2}=v / w(+\infty), w_{2}=$ $w / w(+\infty)$. The proof of Lemma 9.1 of chapter XI of [4] now yields that $\left|w_{2}(\sigma)-1\right| \leqslant C / \sigma^{2}$, and since $v_{2}^{\prime}(\sigma)=\mathrm{e}^{2 \sigma} w_{2}(\sigma)$, we easily obtain 
that $\left|v_{2}(\sigma)-\frac{1}{2} \mathrm{e}^{2 \sigma}\right| \leqslant C \frac{\mathrm{e}^{2 \sigma}}{\sigma^{2}}$. If we define

$$
v_{1}(\sigma)=\left(\int_{\sigma}^{\infty} \mathrm{e}^{2 s}\left(v_{2}(s)\right)^{-2} d s\right) v_{2}(\sigma), \quad w_{1}=\left(w_{2} v_{1}-1\right) / v_{2}
$$

(cf. [4]), simple calculations show that $\left(v_{1}, w_{1}\right)$ is a solution to (B.3) and that $\left|v_{1}(\sigma)-1\right| \leqslant \frac{C}{\sigma^{2}}$ and $\left|w_{1}(\sigma)\right| \leqslant C \frac{e^{-2 \sigma}}{\sigma^{2}}$. If we put $z_{1}(\sigma)=\mathrm{e}^{-\sigma} v_{1}(\sigma)$, $z_{2}(\sigma)=2 \mathrm{e}^{-\sigma} v_{2}(\sigma)$, (B.2) follows easily. Now it is not hard to check that for some $C_{1}, C_{2}>0$, one has $C_{1} \leqslant \mathrm{e}^{\sigma-s / 2} / s^{m} \leqslant C_{2}$ if $s \geqslant \ln 2$. If we use this and define $v_{j}(s)=P^{-1 / 4}(s) z_{j}(\sigma), \zeta_{j}(S)=c_{j} t^{-1 / 2} v_{j}(\ln t), j=1,2$, where $c_{1}, c_{2}$ are suitable strictly positive constants, Lemma 6.1 follows from (B.2) after some straightforward computations.

Proof of Lemma 6.2. - Put

$$
\begin{gathered}
m=\frac{2 \gamma(\gamma+1)}{(\gamma-1)^{2}}, \quad \lambda=\left(m+\frac{1}{4}\right)^{1 / 2}, \quad-Y=\mathrm{e}^{-s}, \\
Z(X, Y)=\mathrm{e}^{-s / 2} v(X, s) .
\end{gathered}
$$

Straightforward computations using Proposition 5.2 show that, for some $s_{0}$,

$$
\partial_{s}^{2} v(X, s)+g_{1}(X, s) \partial_{s} v(X, s)-\left(\lambda^{2}+g_{2}(X, s)\right) v(X, s)=0
$$

if $-\frac{t_{0}}{2} \leqslant X \leqslant b$ and $s \geqslant s_{0}$, where $g_{1}, g_{2} \in C^{1}$ and

$$
\left|g_{1}(X, s)\right|+\mathrm{e}^{(l-1) s}\left|g_{2}(X, s)\right|+\sum_{1 \leqslant j \leqslant 2}\left|\partial_{X} g_{j}(X, s)\right| \leqslant C \mathrm{e}^{-s}
$$

if $-\frac{t_{0}}{2} \leqslant X \leqslant b$ and $s \geqslant s_{0}$, with $l=\min \left(1,2 \frac{\gamma+1}{\gamma-1}\right)$. We shall make use of the following result.

LEMMA B.1. - One can find a fundamental system of solutions $\left\{v_{1}(X\right.$, $\left.s), v_{2}(X, s)\right\}$ of (B.5) such that $v_{1}, v_{2} \in C^{1}\left(\left\{(X, s) \in \mathbb{R}^{2},-\frac{t_{0}}{2} \leqslant X \leqslant\right.\right.$ $\left.\left.b, s \geqslant s_{0}\right\}\right)$ and

$$
\begin{gathered}
\left|\partial_{s}^{j} \nu_{1}(X, s)-(-\lambda)^{j} \mathrm{e}^{-\lambda s}\right| \leqslant C \mathrm{e}^{-(\lambda+l) s}, \quad j=0,1, \\
\left|\partial_{s}^{j} \nu_{2}(X, s)-\lambda^{j} \mathrm{e}^{\lambda s}\right| \leqslant C \mathrm{e}^{(\lambda-l) s}, \quad j=0,1 .
\end{gathered}
$$


Lemma 6.2 easily follows from Lemma B.1 if we put $Z_{k}(X, Y)=$ $\mathrm{e}^{-s / 2} v_{k}(X, s), k=0,1$. Hence it remains to prove Lemma B.1.

Proof of Lemma B.1. - The proof follows the same lines as in Lemma 6.1. Put $v(X, s)=\mathrm{e}^{\lambda s} v(X, s)$. (B.5) can be rewritten as

$$
\begin{gathered}
\partial_{s}\left(\mathrm{e}^{-2 \lambda s} \partial_{s} v(X, s)\right)+g_{1}(X, s)\left(\mathrm{e}^{-2 \lambda s} \partial_{s} v(X, s)\right. \\
\left.-\lambda \mathrm{e}^{-2 \lambda s} v(X, s)\right)-g_{2}(X, s) \mathrm{e}^{-2 \lambda s} v(X, s)=0 .
\end{gathered}
$$

Put

$$
p(X, s)=\exp \left(-\int_{s}^{\infty} g_{1}(X, \sigma) d \sigma\right),
$$

$w(X, s)=\mathrm{e}^{-2 \lambda s} p(X, s) \partial_{s} v(X, s)$. Using (B.6), we obtain

$$
\partial_{s} v(X, s)=\mathrm{e}^{2 \lambda s} A(X, s) w(X, s), \partial_{s} w(X, s)=\mathrm{e}^{-2 \lambda s} B(X, s) v(X, s),
$$

where $A(X, s)=1 / p(X, s), B(X, s)=p(X, s)\left(\lambda g_{1}+g_{2}\right)(X, s)$. In the rest of this proof we shall denote by $C$ various strictly positive constants independent of $X$. Notice that $|B(X, s)| \leqslant C \mathrm{e}^{-l s},\left|A_{X}(X, s)\right|+$ $\left|B_{X}(X, s)\right|+|A(X, s)-1| \leqslant C \mathrm{e}^{-s}$. We may study (B.7) along the same lines as (B.3), the only additional difficulty being the presence of $X$. First we rewrite (B.7) as a system of integral equations

$$
\begin{aligned}
v(X, s)= & \int_{T}^{s} \mathrm{e}^{2 \lambda \sigma} A(X, \sigma) w(X, \sigma) d \sigma+v(X, T), \\
w(X, s)= & \int_{T}^{s} \mathrm{e}^{-2 \lambda \sigma} B(X, \sigma)\left(\int_{T}^{\sigma} \mathrm{e}^{2 \lambda r} A(X, r) w(X, r) d r\right) d \sigma \\
& +v(X, T) \int_{T}^{s} \mathrm{e}^{-2 \lambda \sigma} B(X, \sigma) d \sigma+w(X, T) .
\end{aligned}
$$

Arguing as in the proof of Lemma 6.1, we easily obtain that $w(X, s)$ has a limit $w(X,+\infty)$, uniformly in $X \in\left[-\frac{t_{0}}{2}, b\right]$, as $s \rightarrow+\infty$. Moreover, $|w(X, s)-w(X,+\infty)| \leqslant C \mathrm{e}^{-l s}$. We take $T$ large, $w(X, T) \equiv$ $1, v(X, T) \equiv 0$. Then $|w(X, s)-1| \leqslant \frac{1}{2}$ for all $X \in\left[-\frac{t_{0}}{2}, b\right]$ if $s$ is large. In a similar way, it also follows from (B.8) that $w_{X}(X, s)$ has a limit $\chi(X)$ as $s \rightarrow+\infty$, and that moreover $\left|w_{X}(X, s)-\chi(X)\right| \leqslant$ $C \mathrm{e}^{-l s}$. Hence the function $X \mapsto w(X,+\infty)$ belongs to $C^{1}\left(\left[-\frac{t_{0}}{2}, b\right]\right)$. 
Put $v_{2}(X, s)=v(X, s) / w(X,+\infty), w_{2}(X, s)=w(X, s) / w(X,+\infty)$, $v_{1}(X, s)=\left(\int_{s}^{\infty} \mathrm{e}^{2 \lambda \sigma} \frac{A(X, \sigma)}{v_{2}^{2}(X, \sigma)} d \sigma\right) v_{2}(X, s), w_{1}(X, s)=\frac{w_{2} v_{1}-1}{v_{2}}(X, s)$. Arguing as in the proof of Lemma 6.1, we easily obtain the estimates $\left|w_{2}(X, s)-1\right| \leqslant C \mathrm{e}^{-l s},\left|v_{2}(X, s)-\frac{\mathrm{e}^{2 \lambda s}}{2 \lambda}\right| \leqslant C \mathrm{e}^{(2 \lambda-l) s},\left|v_{1}(X, s)-1\right| \leqslant$ $C \mathrm{e}^{-l s},\left|w_{1}(X, s)\right| \leqslant C \mathrm{e}^{-(2 \lambda+l) s}$, from which Lemma B.1 easily follows.

\section{REFERENCES}

[1] Caffarelli L.A., Friedman A., The blow-up boundary for nonlinear wave equations, Trans. Am. Math. Soc. 297 (1) (1986) 223-241.

[2] Caffarelli L.A., Friedman A., Differentiability of the blow-up curve for one dimensional nonlinear wave equations, Arch. Ration. Mech. Anal. 91 (1985) 83-98.

[3] Godin P., The blow-up curve of solutions of mixed problems for semilinear wave equations with exponential nonlinearities in one space dimension, I, to appear in Calc. Var. Partial Differential Equations.

[4] Hartman P., Ordinary Differential Equations, Wiley, New York, 1964.

[5] Hille E., Lectures on Ordinary Differential Equations, Addison-Wesley, Reading, 1969.

[6] Keller J.B., On solutions of nonlinear wave equations, Comm. Pure Appl. Math. 10 (1957) 523-530.

[7] Kichenassamy S., The blow-up problem for exponential nonlinearities, Comm. Partial Differential Equations 21 (1-2) (1996) 125-162. 